ARTICLE

DOI: $10.1038 / \mathrm{s} 41467-017-01492-6$

\title{
A conformational switch in initiation factor 2 controls the fidelity of translation initiation in bacteria
}

Kelvin Caban ${ }^{1}$, Michael Pavlov², Måns Ehrenberg ${ }^{2}$ \& Ruben L. Gonzalez Jr (i) ${ }^{1}$

Initiation factor (IF) 2 controls the fidelity of translation initiation by selectively increasing the rate of $50 \mathrm{~S}$ ribosomal subunit joining to $30 \mathrm{~S}$ initiation complexes (ICs) that carry an $\mathrm{N}$ formyl-methionyl-tRNA (fMet-tRNA ${ }^{\mathrm{fMet}}$ ). Previous studies suggest that rapid $50 \mathrm{~S}$ subunit joining involves a GTP- and fMet-tRNA ${ }^{\text {fMet }}$-dependent "activation" of IF2, but a lack of data on the structure and conformational dynamics of 30 IC-bound IF2 has precluded a mechanistic understanding of this process. Here, using an IF2-tRNA single-molecule fluorescence resonance energy transfer signal, we directly observe the conformational switch that is associated with IF2 activation within 30S ICs that lack IF3. Based on these results, we

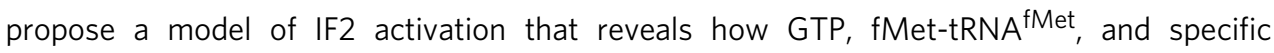
structural elements of IF2 drive and regulate this conformational switch. Notably, we find that domain III of IF2 plays a pivotal, allosteric, role in IF2 activation, suggesting that this domain can be targeted for the development of novel antibiotics.

\footnotetext{
${ }^{1}$ Department of Chemistry, Columbia University, 3000 Broadway, MC3126, New York, NY 10027, USA. ${ }^{2}$ Department of Cell and Molecular Biology, BMC Uppsala University, Husargatan 3, Uppsala 751 24, Sweden. Correspondence and requests for materials should be addressed to

R.L.G. (email: rlg2118@columbia.edu)
} 
nitiation of bacterial protein synthesis, or translation, proceeds along a multi-step pathway that begins with the assembly of a $30 \mathrm{~S}$ initiation complex (IC) (Supplementary Fig. 1a). The $30 \mathrm{~S}$ IC is composed of the small (30S) ribosomal subunit, initiation factor (IF) 1, the guanosine triphosphatase (GTPase) IF2, IF3, initiator $N$-formyl-methionyl-transfer RNA (fMet-tRNA ${ }^{\text {fMet }}$ ), and messenger RNA (mRNA). Although 30S IC assembly can occur via multiple pathways ${ }^{1}$, a kinetically favored pathway has been identified in which the three IFs bind to the 30S subunit and synergistically regulate the kinetics of tRNA binding ${ }^{2}$. Consequently, fMet-tRNA ${ }^{\mathrm{fMet}}$ is preferentially selected into the peptidyl-tRNA-binding $(\mathrm{P})$ site of the $30 \mathrm{~S}$ subunit, where it basepairs to the start codon of an mRNA that can bind to the $30 \mathrm{~S}$ subunit before, during, or after the IFs bind ${ }^{2-7}$. The IFs further enhance the accuracy of translation by cooperatively regulating the rate with which the large (50S) ribosomal subunit joins to the 30 S IC and by modulating the stability of the resulting $70 \mathrm{~S}$ $\mathrm{IC}^{4,5,7-11}$. 70S IC formation triggers GTP hydrolysis by IF2, which subsequently drives a series of maturation steps that enable the 70S IC to enter the elongation stage of protein synthesis ${ }^{12-14}$.

IF2 plays central roles throughout the initiation pathway that ensure accurate fMet-tRNA ${ }^{\mathrm{fMet}}$ selection. During 30S IC assembly, IF2 specifies fMet-tRNA ${ }^{\text {fMet }}$ selection by interacting with the $N$-formyl-methionine and aminoacyl acceptor stem of fMet-

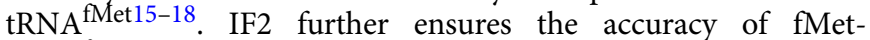
tRNA $^{\text {fMet }}$ selection by preferentially accelerating the rate with which the $50 \mathrm{~S}$ subunit joins to a $30 \mathrm{~S}$ IC carrying fMettRNA ${ }^{\text {fMet } 4,5,9,19-21}$. Indeed, 50S subunit joining to $30 \mathrm{~S}$ ICs carrying GTP-bound IF2 (IF2(GTP)) and P site-bound fMettRNA $^{\text {fMet }}$ is up to between one and two orders of magnitude faster than to 30S ICs in which GTP has been substituted with GDP or to "pseudo" 30S ICs in which fMet-tRNA ${ }^{\text {fMet }}$ has been substituted with an unformylated Met-tRNA ${ }^{\text {fMet }}$, unacylated tRNA $^{\text {fMet }}$, elongator tRNA, or no tRNA at all ${ }^{4,5,19-21}$.

IF2 consists of four conserved structural domains, referred to here as domains I-IV (dI-IV) in the nomenclature of Roll-Mecak et al. ${ }^{22}$, but also referred to as domains G2 (dI), G3 (dII), C1 (dIII), and C2 (dIV) in the nomenclature of Gualerzi et al. ${ }^{23}$ or as domains dIV (dI), dV (dII), dVI-1 (dIII), and dVI-2 (dIV) in the nomenclature of Mortensen et al. ${ }^{24}$ The arrangement of these domains is such that dII and dIII separate the guanine nucleotidebinding domain, dI, from the fMet-tRNA ${ }^{\mathrm{fMet}}$-binding domain, dIV. Structural studies of non-ribosome-associated IF2 strongly suggest that the spatial positions of dIII and dIV are flexible relative to dI and dII, allowing IF2 to adopt increasingly extended conformations upon transitions from nucleotide-free IF2 to IF2 (GDP) and IF2(GTP) ${ }^{25,26}$. Within the context of the 30S IC, dII helps anchor IF2(GTP) to the 30 S IC by interacting with $16 \mathrm{~s}$ ribosomal RNA (rRNA) helices h5 and h14 ${ }^{16}$. Moreover, dIII and $\mathrm{dIV}$ adopt positions relative to $\mathrm{dI}$ and $\mathrm{dII}$ that enable dIV to interact with the $\mathrm{P}$ site-bound fMet-tRNA ${ }^{\text {fMet } 16}$. These interactions, which might be further stabilized by the interactions of dIII with ribosomal protein $S 12^{27}$, result in the formation of an IF2 (GTP)•tRNA sub-complex on the inter-subunit surface of the 30S IC $\mathrm{IC}^{16-18}$.

Previously, Andersson and colleagues ${ }^{20}$ identified IF2 variants containing single amino acid substitution mutations within dIII (mutIF2s) that, remarkably, enable mutIF2(GDP)s to catalyze rapid 50 S subunit joining to 30 S ICs and mutIF2(GTP)s to catalyze rapid $50 \mathrm{~S}$ subunit joining to pseudo $30 \mathrm{~S} \mathrm{ICs}^{20,21}$. Based on these results, we have proposed that IF2 is "activated" for rapid $50 \mathrm{~S}$ subunit joining by a GTP- and fMet-tRNA ${ }^{\text {fMet }}$-dependent conformational switch that is rendered GTP- and fMettRNA $^{\text {fMet }}$-independent by the "activating" mutations in dIII of the mutIF2s ${ }^{20,21}$. Nonetheless, due to a lack of experimental data on the structure of IF2(GDP)-bound 30S ICs, IF2(GTP)-bound a

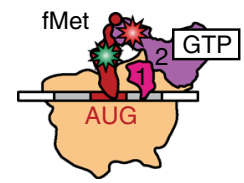

b
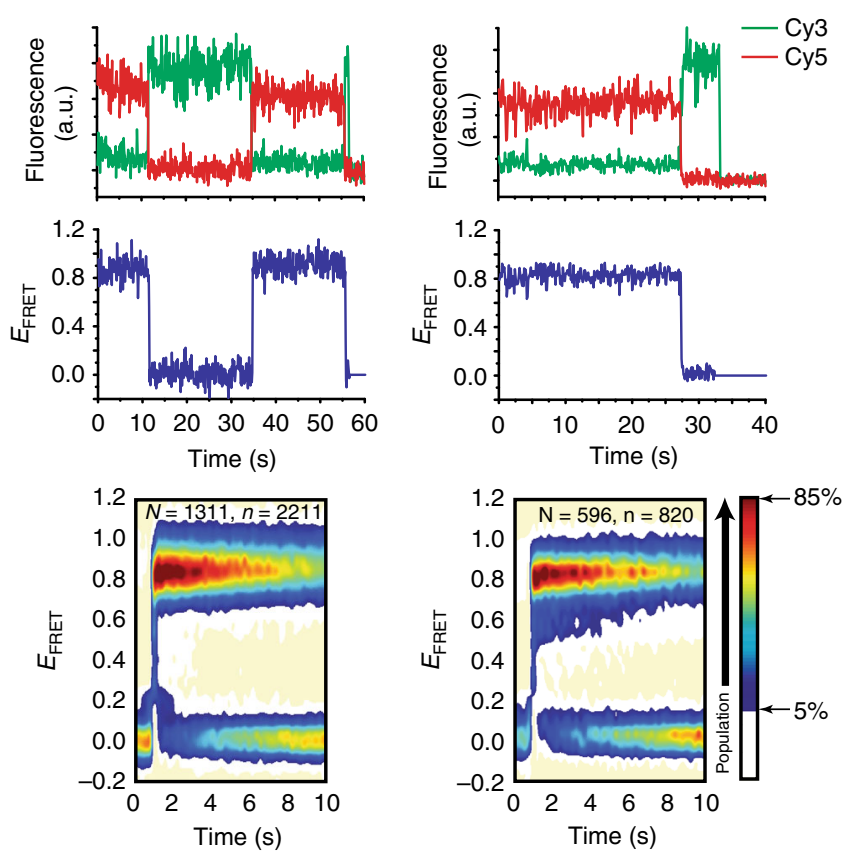

Fig. 1 Effect of GTP and fMet-tRNA ${ }^{\mathrm{fMet}}$. smFRET measurements of (a) wtIF2(GTP) and (b) mutIF2(GTP) interacting with $30 \mathrm{SIC}_{\mathrm{wT}}$ and $30 \mathrm{~S}$ $I C_{m T}$, respectively. First row: cartoon illustrations depicting $30 S I C_{W T}$-bound wtIF2(GTP) (light purple) and $30 \mathrm{~S} \mathrm{IC}_{\mathrm{mT}}$-bound mutIF2(GTP) (dark purple). Second row: representative $\mathrm{Cy} 3$ (green) and Cy5 (red) emission intensities vs. time trajectories. Third row: corresponding $E_{\text {FRET }}$ vs. time trajectories. Fifth row: post-synchronized surface contour plots of the time evolution of population FRET. Surface contour plots were generated by superimposing hundreds of individual IF2-binding events. " $N$ " indicates the total number of $E_{\text {FRET }}$ VS. time trajectories for each $30 \mathrm{SIC}$ and " $n$ " indicates the total number of individual IF2-binding events. The surface contours were plotted from tan (lowest population plotted) to red (highest population plotted) as indicated in the population color bar

pseudo 30S ICs, mutIF2(GDP)-bound 30S ICs, and/or mutIF2 (GTP)-bound pseudo 30S ICs, as well as on the GTP- and fMet-

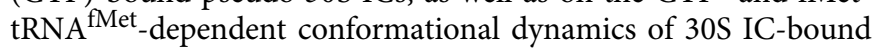
IF2 and mutIF2, the structural basis and molecular mechanism of IF2 activation have remained unknown.

To close this gap in our understanding of how IF2 helps regulate the fidelity of translation initiation, here we report an investigation of the structural dynamics of GTP- and fMet-

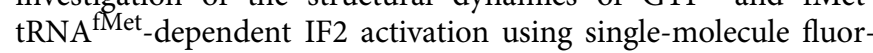
escence resonance energy transfer (smFRET). Our data provide direct evidence that IF2 activation consists of a conformational switch of IF2 and demonstrate that the GTP- and fMettRNA $^{\text {fMet }}$-dependent dynamics of this switch regulates IF2 activation by modulating the affinity of IF2 for the 30 S IC and the conformation of 30 S IC-bound IF2. Based on these results, we propose a model for IF2 activation specifying how GTP, fMet$\mathrm{tRNA}^{\mathrm{fMet}}$, and the four domains of IF2 collectively drive and regulate the dynamics of this conformational switch. Interestingly, we find that dIII allosterically regulates IF2 activation, 

Table 1 Association rate constant $\left(k_{\mathrm{a}}\right)$, dissociation rate constant $\left(k_{\mathrm{d}}\right)$, and dissociation equilibrium constant $\left(K_{\mathrm{d}}\right)$ for the
interaction of IF 2 and the 30 S IC

\begin{tabular}{|c|c|c|c|c|c|}
\hline $30 \mathrm{~S} \mathrm{IC}$ & IF2 & Nucleotide & $k_{\mathrm{a}}\left(\mu \mathrm{M}^{-1} \mathrm{~s}^{-1}\right)^{\mathrm{a}}$ & $k_{\mathrm{d}}\left(\mathrm{s}^{-1}\right)^{\mathrm{a}}$ & $K_{\mathrm{d}}(\mathrm{nM})^{\mathrm{a}}$ \\
\hline $\begin{array}{l}30 \mathrm{~S} I C_{\mathrm{wD}} \\
30 \mathrm{~S} I C_{\mathrm{mD}}\end{array}$ & $\begin{array}{l}\text { wtIF2 } \\
\text { mutIF2 }\end{array}$ & $\begin{array}{l}\text { GDP } \\
\text { GDP }\end{array}$ & $\begin{array}{l}2.1 \pm 0.12 \\
1.2 \pm 0.10\end{array}$ & $\begin{array}{l}1.32 \pm 0.09 \\
0.13 \pm 0.01\end{array}$ & $\begin{array}{l}622 \pm 28 \\
102 \pm 9\end{array}$ \\
\hline $\begin{array}{l}30 S I_{\mathrm{wT}, \text { Met }} \\
30 \mathrm{~S} I C_{\mathrm{mT}, \text { Met }}\end{array}$ & $\begin{array}{l}\text { wtIF2 } \\
\text { mutIF2 }\end{array}$ & $\begin{array}{l}\text { GTP } \\
\text { GTP }\end{array}$ & $\begin{array}{l}0.52 \pm 0.02^{c} \\
0.77 \pm 0.05\end{array}$ & $\begin{array}{l}1.2 \pm 0.2 \\
0.11 \pm 0.01\end{array}$ & $\begin{array}{l}2328 \pm 294 \\
136 \pm 7\end{array}$ \\
\hline
\end{tabular}

${ }^{a} k_{a}, k_{d}$, and $K_{d}$ were obtained from three independently collected data sets (mean $\pm \mathrm{SE}$ ) using a transition probability matrix-based population decay analysis as described previously ${ }^{28}$ and in Methods section $b_{k_{a}}$ and $k_{d}$ were corrected for the effects of Cy5 photobleaching

${ }^{c} k_{a}$ was corrected for the effects of $C y 3$ photobleaching

highlighting dIII as an attractive target for the development of novel antibiotics that function as allosteric inhibitors of IF2.

\section{Results}

Escherichia coli mutIF2 catalyzes rapid 50S subunit joining. mutIF2s were initially selected in Salmonella (S) typhimurium on the basis of their ability to complement the slow growth phenotype arising from a Met-tRNA ${ }^{\text {fMet }}$ formylation deficiency ${ }^{20}$. One such $S$. typhimurium mutIF2 contains a Ser755Tyr mutation in dIII and has been shown to strongly compensate for a Met-tRNA ${ }^{\text {fMet }}$ formylation deficiency both in vivo and in vitro ${ }^{20,21}$. Here, we generated the homologous E. coli Ser753Tyr mutIF2 (Supplementary Fig. 2), purified it, and confirmed its ability to catalyze rapid $50 \mathrm{~S}$ subunit joining to both 30 S ICs and pseudo 30S ICs using ensemble kinetic studies of subunit joining (Supplementary Fig. 3). Importantly, a Gly810Cys mutation in dIV, previously used to label E. coli IF2 with a FRET acceptor fluorophore (ref. ${ }^{28}$ and vide infra), did not alter the kinetic performance of either E. coli IF2(GTP) or E. coli Ser753Tyr mutIF2(GTP). We further validated the biochemical activities of our unlabeled IF2 variants using a standard, biochemical IF2 activity assay that is based on primer extension inhibition, or "toeprinting" (Supplementary Fig. 4). Unless otherwise specified, the designations "wtIF2" and "mutIF2" will hereafter refer to E. coli wild-type IF2 and E. coli Ser753Tyr mutIF2, respectively, both harboring an additional Gly810Cys mutation in dIV.

wtIF2(GTP) and mutIF2(GTP) adopt similar conformations. To characterize the interaction of wtIF2 and mutIF2 with 30S ICs and pseudo 30S ICs, we used a previously developed IF2-tRNA smFRET signal ${ }^{28}$. This signal reports on changes in the distance between a cyanine 5 (Cy5) FRET acceptor fluorophore in dIV of IF2 (wtIF2 [Cy5 $]_{\mathrm{dIV}}$ or mutIF2[Cy5 $\left.]_{\mathrm{dIV}}\right)$ and a cyanine 3 (Cy3) FRET donor fluorophore in the central fold, or "elbow", domain of $\mathrm{tRNA}^{\mathrm{fMet}}\left(\mathrm{tRNA}(\mathrm{Cy} 3)^{\mathrm{fMet}}\right)$, thereby reporting on the formation and conformational dynamics of the IF2•tRNA sub-complex (Supplementary Fig. 1b). We began by assembling a 30S IC using $30 \mathrm{~S}$ subunits, a 5'-biotinylated mRNA, fMet-tRNA $(\mathrm{Cy} 3)^{\mathrm{fMet}}$, IF1, wtIF2 $[\mathrm{Cy} 5]_{\mathrm{dIV}}$, and GTP (hereafter referred to as $30 \mathrm{~S} \mathrm{IC}_{\mathrm{wT}}$, where the "w" and "T" subscripts denote wtIF2[Cy5 $]_{\text {dIV }}$ and GTP, respectively). Previously, we have shown that IF3 destabilizes the binding of all tRNAs to the $30 \mathrm{~S}$ subunit P site ${ }^{4,5,29}$; thus, IF3 was excluded from the assembly of all of the 30 S ICs and pseudo $30 \mathrm{~S}$ ICs in the current study. We note that, even in the absence of IF3, IF2 retains the ability to selectively accelerate the rate of $50 \mathrm{~S}$ subunit joining to correctly assembled $30 \mathrm{~S} \mathrm{ICs}^{4,5,21}$. Furthermore, exclusion of IF3 provides a simple model system to allow for clarification of the basal conformational changes of 30S IC-bound
IF2 that confer rapid and selective $50 \mathrm{~S}$ subunit joining. Following previously published protocols ${ }^{28}, 30 \mathrm{~S}_{\mathrm{IC}}$ w was then tethered to the surface of a quartz microfluidic flowcell and imaged at singlemolecule resolution using a total internal reflection fluorescence (TIRF) microscope operating at an acquisition time of $0.1 \mathrm{~s}$ per frame. As before ${ }^{28}$, we supplemented all buffers with $25 \mathrm{nM}$ wtIF2 $[\mathrm{Cy} 5]_{\mathrm{dIV}}(\mathrm{GTP})$ in order to allow re-association of wtIF2 $[\mathrm{Cy} 5]_{\mathrm{dIV}}(\mathrm{GTP})$ with $30 \mathrm{~S} \mathrm{IC}_{\mathrm{wT}} \mathrm{s}$ from which it might have dissociated during tethering and/or TIRF imaging.

Consistent with our previous smFRET studies $^{28}$, individual FRET efficiency $\left(E_{\mathrm{FRET}}\right)$ vs. time trajectories exhibited reversible fluctuations between a zero FRET state, corresponding to the IF2free state of $30 \mathrm{~S}_{\mathrm{IC}}$, and a non-zero FRET state, corresponding to the wtIF2(GTP)-bound state of $30 \mathrm{~S} \mathrm{IC}_{\mathrm{wT}}$ (Fig. 1a). Kinetic and thermodynamic parameters describing the interaction of wtIF2 (GTP) with $30 \mathrm{~S} \mathrm{IC}_{\mathrm{wT}}$ were determined using previously described methods (see ref. ${ }^{28}$ and Methods section). Briefly, we learned a hidden Markov model (HMM) from the $E_{\mathrm{FRET}}$ trajectories to determine the probabilities of transitioning between the IF2-freeand wtIF2(GTP)-bound states of $30 \mathrm{~S} \mathrm{IC}_{\mathrm{wT}}$ and converted the resulting state transition probabilities into rate constants using a transition probability matrix-based population decay analysis. Using this approach, we determined the bimolecular rate constant for the association of wtIF2(GTP) to $30 \mathrm{SIC}_{\mathrm{wT}}\left(k_{\mathrm{a}, \mathrm{wT}}\right)$ to be $2.0 \pm$ $0.1 \mu \mathrm{M}^{-1} \mathrm{~s}^{-1}$, the rate constant for the dissociation of wtIF2(GTP) from $30 \mathrm{~S} \mathrm{IC}_{\mathrm{wT}}\left(k_{\mathrm{d}, \mathrm{wT}}\right)$ to be $0.041 \pm 0.01 \mathrm{~s}^{-1}$, and the equilibrium dissociation constant for the wtIF2(GTP)-30S IC $_{\mathrm{wT}}$ complex $\left(K_{\mathrm{d}, \mathrm{wT}}\right)$ to be $21 \pm 6 \mathrm{nM}$ (Table 1$)$.

To characterize the conformational dynamics of the wtIF2(GTP) tRNA sub-complex on $30 S \mathrm{IC}_{\mathrm{wT}}$, we plotted histograms of the $E_{\mathrm{FRET}}$ values observed for the wtIF2(GTP)-bound state of $30 \mathrm{~S} \mathrm{IC}_{\mathrm{wT}}$ (Fig. 1a and Supplementary Fig. 5a). The distribution of $E_{\text {FRET }}$ values exhibited a single non-zero $E_{\mathrm{FRET}}$ peak that was centered at a mean $E_{\text {FRET }}$ value $\left(\left\langle E_{\text {FRET }}\right\rangle\right.$ ) of $0.87 \pm 0.02$ (Supplementary Table 1). Using a Förster distance $\left(R_{0}\right)$ of $55 \AA$ for the Cy3-Cy5 FRET donor-acceptor pair ${ }^{30}$ and assuming unrestricted, isotropic motion of the fluorophores, this $\left\langle E_{\mathrm{FRET}}>\right.$ corresponds to an $\sim 40 \AA$ average separation between our labeling positions, a separation that is consistent with the cryogenic electron microscopy (cryo-EM) structure of 30 S IC-bound IF2(GTP) ${ }^{18}$.

To investigate the effects that the activating mutation in dIII has on the affinity of IF2(GTP) for the 30S IC and the conformation of 30S IC-bound IF2(GTP), we performed smFRET experiments using mutIF2[Cy5 $]_{\mathrm{dIV}}(\mathrm{GTP})$ and $30 \mathrm{~S} \mathrm{IC}_{\mathrm{mT}}$ (where the " $m$ " subscript denotes mutIF2[Cy5 $\left.]_{\mathrm{dIV}}\right)$. The results demonstrate that excursions to the mutIF2(GTP)-bound state in the $30 \mathrm{~S}$ $\mathrm{IC}_{\mathrm{mT}} E_{\mathrm{FRET}}$ trajectories are longer-lived than those to the wtIF2 (GTP)-bound state in the $30 \mathrm{~S} \mathrm{IC}_{\mathrm{wT}} E_{\mathrm{FRET}}$ trajectories (compare 
Fig. 1a and b). Consistent with this, $K_{\mathrm{d}, \mathrm{mT}}$ is approximately threefold smaller than $K_{\mathrm{d}, \mathrm{wT}}$ (Table 1), demonstrating that the activating mutation confers a higher affinity of mutIF2(GTP) for $30 \mathrm{~S} \mathrm{IC}_{\mathrm{mT}}$ than the affinity of wtIF2(GTP) for $30 \mathrm{~S} \mathrm{IC}_{\mathrm{wT}}$. Interestingly, the distribution of $E_{\mathrm{FRET}}$ values for the mutIF2 (GTP)-bound state of $30 \mathrm{~S} \mathrm{IC}_{\mathrm{mT}}$ (Fig. $1 \mathrm{~b}$ and Supplementary Fig. 5b) was composed of a single non-zero $E_{\text {FRET }}$ peak that was centered at an $\left\langle E_{\mathrm{FRET}}\right\rangle$ of $0.85 \pm 0.01$ that is within error of that observed for the wtIF2(GTP)-bound state of $30 \mathrm{~S} \mathrm{IC}_{\mathrm{wT}}$ ( $p$ value $=$ 0.2 , Supplementary Table 1$)$. This indicates that the conformation of $30 \mathrm{~S} \mathrm{IC}_{\mathrm{mT}^{-}}$-bound mutIF2(GTP) is not significantly altered by the activating mutation and is very similar to that of a $30 \mathrm{~S}$ $\mathrm{IC}_{\mathrm{wT}}$-bound wtIF2(GTP). Previously, we have used ensemble kinetic experiments to show that wtIF2(GTP) and mutIF2(GTP) can catalyze rapid $50 \mathrm{~S}$ subunit joining to $30 \mathrm{~S} \mathrm{IC}_{\mathrm{wT}}{ }^{*}$ and $30 \mathrm{~S}$ $\mathrm{IC}_{\mathrm{mT}}{ }^{*}$ (where the asterisk denotes the analogous 30S IC in the kinetic studies) $)^{4,5,20,21}$. We therefore interpret the observed $\left\langle E_{\mathrm{FRET}}\right\rangle$ s of $\sim 0.85$ and $\sim 0.87$ as corresponding to a conformation of the IF2(GTP)•tRNA sub-complex in which IF2(GTP) is active for rapid $50 \mathrm{~S}$ subunit joining.

GTP allosterically positions dIV closer to the P-site tRNA. We next performed smFRET experiments using wtIF2[Cy5 $]_{\mathrm{dIV}}(\mathrm{GDP})$ and $30 \mathrm{~S}_{\mathrm{wD}}$ (where the "D" subscript denotes GDP) to explore if and how the affinity of IF2 for the 30 S IC and the conformation of 30 S IC-bound IF2 depend on the guanine nucleotide that is bound to IF2. These experiments reveal that excursions to the wtIF2(GDP)-bound state in the $30 \mathrm{~S} \mathrm{IC}_{\mathrm{wD}} E_{\mathrm{FRET}}$ trajectories are more transient than those to the wtIF2(GTP)-bound state in the $30 S \mathrm{IC}_{\mathrm{wT}} E_{\mathrm{FRET}}$ trajectories (compare Figs. 2a and 1a). Correspondingly, we observe a $K_{\mathrm{d}, \mathrm{wD}}$ value that is $\sim 30$-fold larger than the $K_{\mathrm{d}, \mathrm{wT}}$ value (Table 1 ), demonstrating that the affinity of wtIF2 binding to the $30 \mathrm{~S}$ IC is much higher when GTP, rather than GDP, is bound to IF2. In addition, we found that the distribution of $E_{\mathrm{FRET}}$ values for the wtIF2(GDP)-bound state of $30 \mathrm{~S} \mathrm{IC}_{\mathrm{wD}}$ (Fig. 2a and Supplementary Fig. 5c) exhibited two non-zero $E_{\mathrm{FRET}}$ peaks. One of the peaks encompassed a minor, $18 \pm 1.5 \%$, subpopulation of $30 \mathrm{~S}_{\mathrm{ICD}_{\mathrm{w}}}$-bound wtIF2(GDP) and was centered at an $\left\langle E_{\mathrm{FRET}}\right\rangle$ of $0.89 \pm 0.01$ that is within error of that observed for $30 \mathrm{~S} \mathrm{IC}_{\mathrm{wT}}$-bound wtIF2(GTP) ( $p$ value $=0.2$, Supplementary Table 1$)$. The other peak encompassed a major, $82 \pm 1.5 \%$, subpopulation of $30 \mathrm{~S} \mathrm{IC}_{\mathrm{wD}}$-bound wtIF2(GDP) and was centered at an $\left\langle E_{\mathrm{FRET}}\right\rangle$ of $0.67 \pm 0.01$ that is notably lower than that observed for $30 \mathrm{~S} \mathrm{IC}_{\mathrm{wT}}$-bound wtIF2(GTP) ( $p$ value $=0.002$, Supplementary Table 1).

Previously, we have used ensemble kinetic experiments to show that $30{\mathrm{~S} \mathrm{IC}_{\mathrm{wD}}}^{*}$ exhibits a drastic, $\sim 60$-fold smaller rate of $50 \mathrm{~S}$ subunit joining than $30 \mathrm{~S} \mathrm{IC}_{\mathrm{wT}}{ }^{* 21}$. Based on the values of $K_{\mathrm{d}, \mathrm{wD}}$ and $K_{\mathrm{d}, \mathrm{wT}}$ determined here $(622 \mathrm{nM}$ and $21 \mathrm{nM}$, respectively) and the IF2 and 30S IC concentrations employed in our previous kinetic studies of $50 \mathrm{~S}$ subunit joining 21 , we estimate that the occupancy of wtIF2(GDP) on $30 \mathrm{~S}_{\mathrm{wD}}{ }^{*}$ in our previous studies was only twofold lower than the occupancy of wtIF2(GTP) on $30 S \mathrm{IC}_{\mathrm{wT}}{ }^{*}$ (Supplementary Table 2). Thus, this occupancy difference is insufficient to account for the decreased rate of $50 \mathrm{~S}$ subunit joining to $30 \mathrm{~S}_{\mathrm{IC}_{\mathrm{wD}}}{ }^{\star}$. Instead, we conclude that the decreased rate of $50 \mathrm{~S}$ subunit joining primarily arises from the stabilization of a major subpopulation of $30 \mathrm{~S} \mathrm{IC}_{\mathrm{wD}}$-bound wtIF2 (GDP) in a conformation that is inactive for rapid 50S subunit joining and that, given our measured $\left\langle E_{\mathrm{FRET}}\right\rangle$ values, features a separation between our labeling positions that is $\sim 9 \AA$ longer than what it is in a $30 \mathrm{~S} \mathrm{IC}_{\mathrm{wT}}$-bound wtIF2(GTP) that is active for rapid $50 \mathrm{~S}$ subunit joining. Given that $\mathrm{dIV}$ is connected to dIII via a potentially flexible linker ${ }^{34}$, this $\sim 9 \AA$ increase in the distance between $\mathrm{dIV}$ and the P-site tRNA can arise from two different a
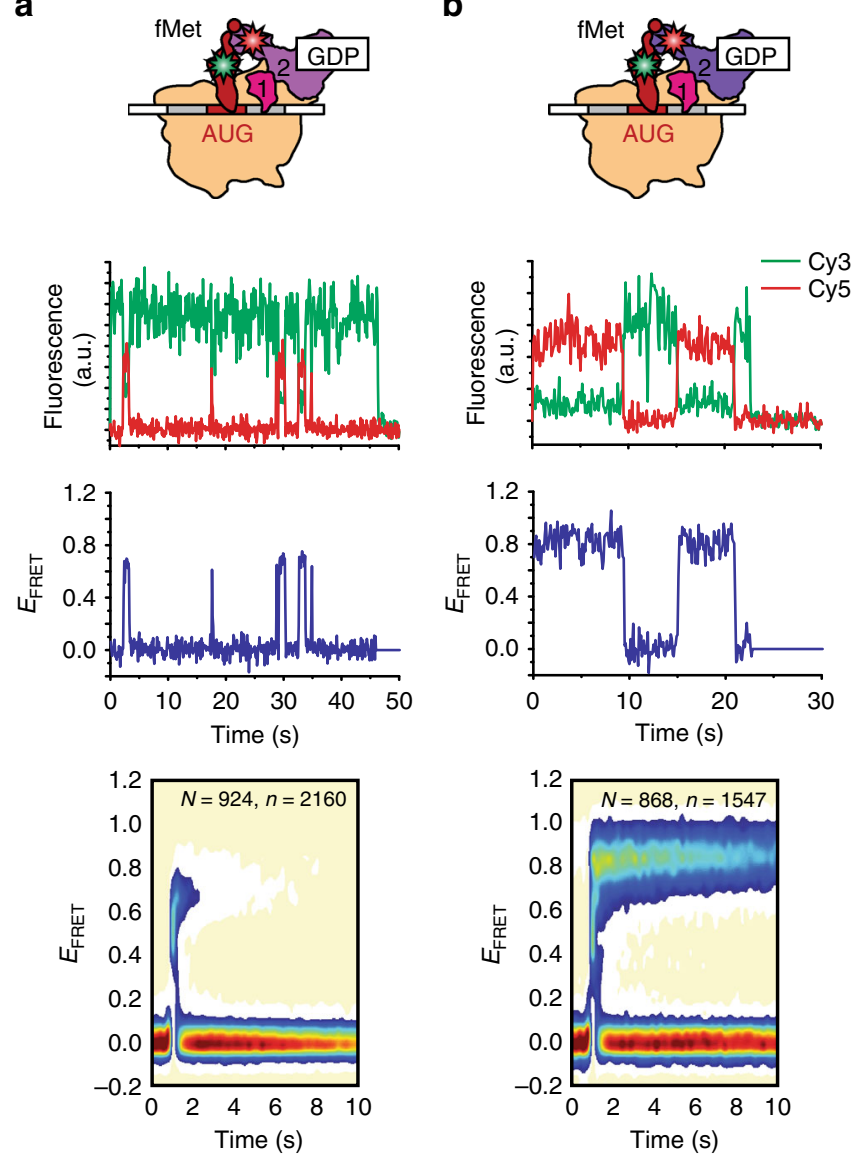

Fig. 2 Effect of substituting GTP with GDP. smFRET measurements of (a) wtIF2(GDP) and (b) mutIF2(GDP) interacting with $30 S I_{\mathrm{wD}}$ and $30 \mathrm{~S}$ $I C_{\mathrm{mD}}$, respectively. Data are displayed as in Fig. 1

scenarios. In the first scenario, dIV adopts a single, fixed position that is $\sim 49 \AA$ from the P-site tRNA. In the alternative scenario, dIV adopts multiple positions that interconvert on a timescale that is faster than the acquisition time of our TIRF microscope (i.e., $0.1 \mathrm{~s}$ per frame), yielding a time-averaged position that is $\sim 49 \AA$ from the P-site tRNA.

Such a difference between the conformations of the GDP- and GTP-bound forms of IF2 is consistent with comparative structural analyses of non-ribosome-associated IF2(GDP) and IF2(GTP) ${ }^{26}$ and of $70 S$ IC-bound IF2(GDP) ${ }^{31}$ and IF2(GTP) ${ }^{27,31-33}$. Based on these analyses, we propose that the guanine nucleotide bound to $\mathrm{dI}$ of $30 \mathrm{~S}$ IC-bound IF2 allosterically modulates the position of dIV relative to that of dI-dIII and the P-site tRNA. Indeed, compared to the position of dIV in IF2(GDP) in these structures, dIV in IF2(GTP) is positioned further away from dI-dIII and closer to the P-site tRNA.

To validate this model, we developed a wtIF2 variant in which dIII was labeled with a Cy5 fluorophore (wtIF2[Cy5 $]_{\text {dIII }}$ ), Methods section), and used wtIF2[Cy5 $]_{\text {dIII }}$ to repeat the smFRET experiments described above. We found that the distributions of $E_{\text {FRET }}$ values for the wtIF2(GTP)-bound state of 30 S $_{\text {wT }}$ and the wtIF2(GDP)-bound state of $30 \mathrm{~S} \mathrm{IC}_{\mathrm{wD}}$ were both composed of only a single non-zero $E_{\mathrm{FRET}}$ peak that was centered at an $\left\langle E_{\mathrm{FRET}}>\right.$ of $\sim 0.3$ and an average distance between our labeling positions of $\sim 63 \AA$ (Supplementary Fig. 6). These results strongly suggest that the relative distance between $\mathrm{dIII}$ and the P-site tRNA is similar in the minor and major subpopulations of $30 \mathrm{~S}$ $\mathrm{IC}_{\mathrm{wD}}$-bound wtIF2(GDP) and that this distance is comparable to the corresponding distance in $30 \mathrm{~S} \mathrm{IC}_{\mathrm{wT}}$-bound wtIF2(GTP). 
a
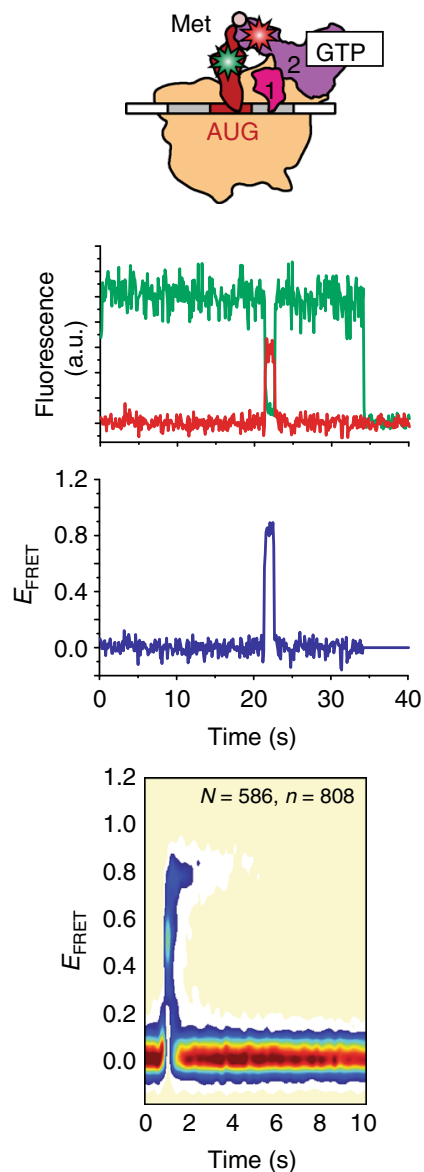

b
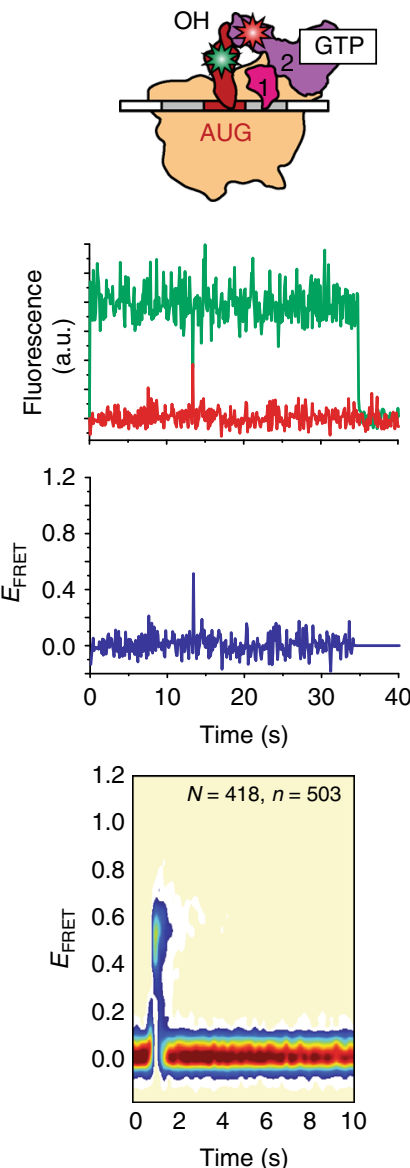

C
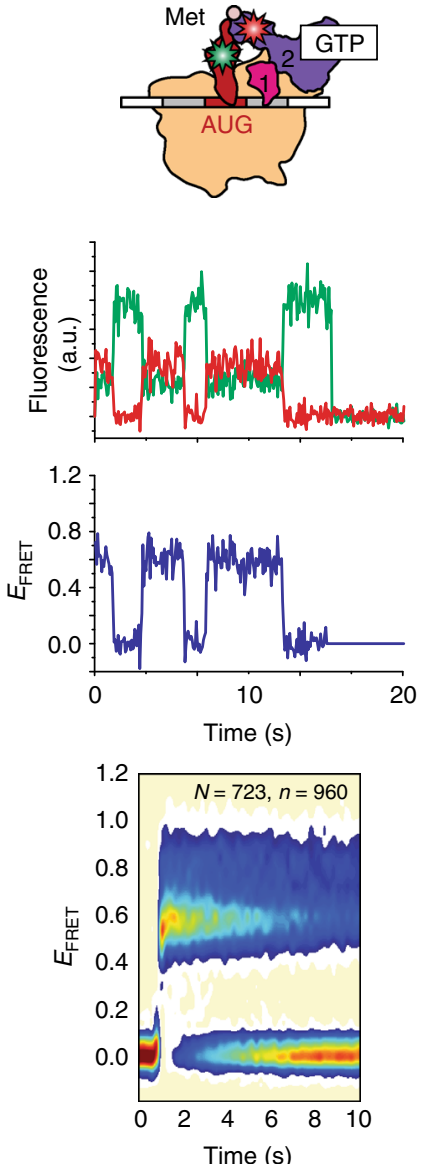

d
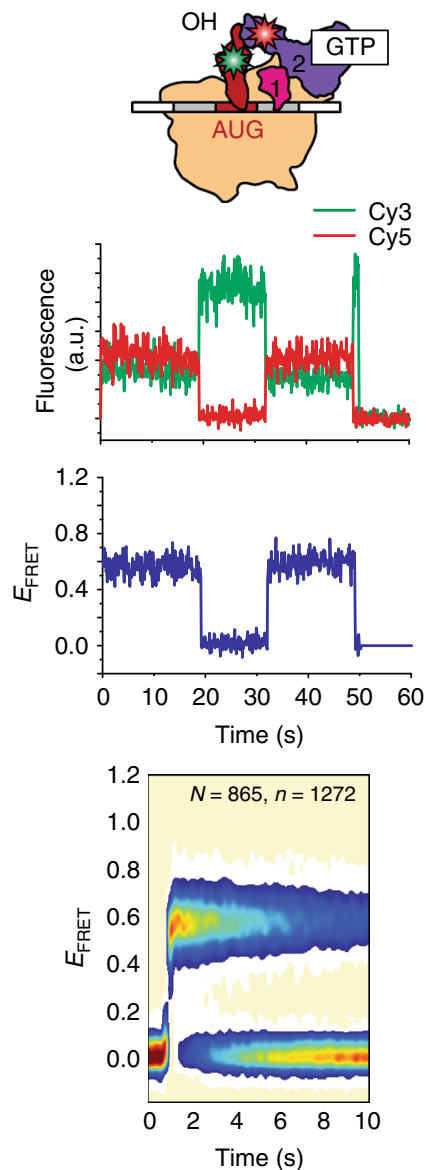

Fig. 3 Effect of substituting fMet-tRNA ${ }^{\text {fMet }}$ with Met-tRNA ${ }^{\text {fMet }}$ or tRNA ${ }^{\text {fMet }}$. smFRET measurements of (a, b) wtIF2(GTP) and (c, d) mutIF2(GTP)

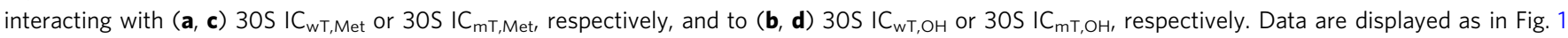

Notably, however, we were able to unambiguously identify two kinetically distinguishable subpopulations of the wtIF2(GDP)bound state of $30 \mathrm{~S} \mathrm{IC}_{\mathrm{wD}}$ whose kinetic properties were equivalent to those of the minor and major subpopulations of the wtIF2 (GDP)-bound state of $30 \mathrm{~S} \mathrm{IC}_{\mathrm{wD}}$ that we identified using wtIF2 $[\mathrm{Cy} 5]_{\mathrm{dIV}}$ (Supplementary Fig. 7). Collectively, the data obtained using wtIF2 $[\mathrm{Cy} 5]_{\mathrm{dIII}}$ and wtIF2[Cy5 $]_{\mathrm{dIV}}$ allows us to validate and extend the structural model described above.

The activating mutation in dIII allosterically positions dIV. To determine whether and how the activating mutation in dIII modulates the affinity of IF2(GDP) for the 30S IC and the conformation of $30 \mathrm{~S}$ IC-bound IF2(GDP), we performed smFRET experiments using mutIF2[Cy5 $]_{\mathrm{dIV}}(\mathrm{GDP})$ and $30 \mathrm{~S} \mathrm{IC}_{\mathrm{mD}}$. The results show that excursions to the mutIF2(GDP)-bound state in the $30 \mathrm{~S} \mathrm{IC}_{\mathrm{mD}} E_{\mathrm{FRET}}$ trajectories are significantly longer than those to the wtIF2(GDP)-bound state in the $30 \mathrm{SC}_{\mathrm{wD}} E_{\mathrm{FRET}}$ trajectories (compare Fig. 2a and b). In line with this, we find that the value of $K_{\mathrm{d}, \mathrm{mD}}$ is approximately sixfold smaller than that of $K_{\mathrm{d}, \mathrm{wD}}$ (Table 1). Thus, the activating mutation in dIII enables mutIF2 (GDP) to bind to $30 \mathrm{~S}_{\mathrm{IC}_{\mathrm{mT}}}$ with a higher affinity than wtIF2 (GDP) binds to $30 \mathrm{~S}_{\mathrm{IC}_{\mathrm{wD}}}$. More importantly, however, we find that the distribution of $E_{\mathrm{FRET}}$ values for the mutIF2(GDP)-bound state of $30 \mathrm{~S}_{\mathrm{mD}}$ (Fig. $2 \mathrm{~b}$ and Supplementary Fig. $5 \mathrm{~d}$ ) is composed of a single non-zero $E_{\mathrm{FRET}}$ peak centered at an $\left\langle E_{\mathrm{FRET}}\right\rangle$ of $0.86 \pm 0.03$ that is within error of that observed for the wtIF2 (GTP)-bound state of $30 \mathrm{~S} \mathrm{IC}_{\mathrm{wT}}$ ( $p$ value $=0.8$, Supplementary Table 1). Thus, remarkably, the activating mutation in dIII enables $30 \mathrm{~S} \mathrm{IC}_{\mathrm{mD}}$-bound mutIF2(GDP) to adopt a conformation that closely resembles that observed for a $30 \mathrm{~S} \mathrm{IC}_{\mathrm{wT}}$-bound wtIF2 (GTP) that is active for rapid 50S subunit joining.

Previously, we have used ensemble kinetic experiments to show that the rate of $50 \mathrm{~S}$ subunit joining to $30 \mathrm{~S} \mathrm{IC}_{\mathrm{mD}}{ }^{*}$ is $\sim 40$-fold higher than to $30 \mathrm{~S}_{\mathrm{IC}_{\mathrm{wD}}}{ }^{21}$. Thus, the activating mutation in dIII enables mutIF2(GDP) to catalyze $50 \mathrm{~S}$ subunit joining to $30 \mathrm{~S}$ $\mathrm{IC}_{\mathrm{mD}}{ }^{*}$ at a rate similar to that observed for $50 \mathrm{~S}$ subunit joining to $30 \mathrm{~S}_{\mathrm{IC}_{\mathrm{wT}}}{ }^{*}$. Based on the results reported here, we propose that the activating mutation in $\mathrm{dIII}$ enables rapid $50 \mathrm{~S}$ subunit joining by stabilizing a conformation of dI-dIII that increases the affinity of mutIF2(GDP) for $30 \mathrm{~S}_{\mathrm{mD}}$ and that enables mutIF2(GDP) to position dIV closer to the $P$ site such that it can interact with the $\mathrm{P}$ site-bound fMet-tRNA ${ }^{\mathrm{fMet}}$. Moreover, our result also implies that stabilizing the analogous conformation of $30 \mathrm{~S}$ IC-bound wtIF2 requires the binding of GTP to $\mathrm{dI}$. Hence, it is the conformation of dI-dIII, which in wtIF2 is specified by the guanine nucleotide that is bound to $\mathrm{dI}$, that determines the position of dIV relative to the P-site tRNA and controls the activation of 30 S IC-bound IF2 for rapid 50S subunit joining.

fMet-tRNA ${ }^{\text {fMet }}$ stabilizes the active conformation of wtIF2. Next, we performed smFRET experiments using wtIF2 [Cy5 $]_{\mathrm{dIV}}(\mathrm{GTP})$ and analogs of $30 \mathrm{~S} \mathrm{IC}_{\mathrm{wT}}$ in which the fMettRNA ${ }^{\text {fMet }}$ has been substituted with Met-tRNA ${ }^{\text {fMet }}$ (30S IC $_{\text {wT,Met }}$ ) or $\mathrm{tRNA}^{\mathrm{fMet}}\left(30 \mathrm{~S} \mathrm{IC}_{\mathrm{wT}, \mathrm{OH}}\right)$ to investigate if and how the affinity of IF2 for the 30S IC and the conformation of 30S IC-bound IF2 depend on the $\mathrm{N}$-formyl moiety and/or methionine of the $30 \mathrm{~S}$ IC- 
bound fMet-tRNA ${ }^{\mathrm{fMet}}$. Consistent with our previous smFRET studies $^{28}$, we found that the $30 \mathrm{~S} \mathrm{IC}_{\mathrm{wT}, \mathrm{Met}}$ and $30 \mathrm{~S} \mathrm{IC}_{\mathrm{wT}, \mathrm{OH}} E_{\mathrm{FRET}}$ trajectories exhibit excursions to the wtIF2(GTP)-bound state that are much shorter lived than those of $30 \mathrm{~S} \mathrm{IC}_{\mathrm{wT}}$ (compare Fig. 3a, b with Fig. 1a). In line with this, $K_{\text {d,wT,Met }}$ is $\sim 100$-fold and $K_{\mathrm{d}, \mathrm{wT}, \mathrm{OH}}$ is $\sim 300$-fold larger than $K_{\mathrm{d}, \mathrm{wT}}$ (Table 1 ). These results suggest that the absence of just the $N$-formyl moiety or the $N$ formyl-methionine from the $30 \mathrm{~S}$ IC-bound fMet-tRNA ${ }^{\text {fMet }}$ is enough to disrupt interactions between dIV and fMet-tRNA ${ }^{\text {fMet }}$ that significantly contribute to anchoring wtIF2(GTP) to the $30 \mathrm{~S}$ IC.

Consistent with our previous smFRET studies ${ }^{28}$, we find that the distribution of $E_{\mathrm{FRET}}$ values for the wtIF2(GTP)-bound state of $30 \mathrm{~S} \mathrm{IC}_{\mathrm{wT}, \mathrm{Met}}$ (Fig. 3a and Supplementary Fig. 5e) is very broad, with values in the $0.2-1.0$ range that encompass two non-zero $E_{\text {FRET }}$ peaks. The peak corresponding to the larger, $56 \pm 12 \%$, subpopulation of $30 \mathrm{~S} \mathrm{IC}_{\mathrm{wT}, \mathrm{Met}}$-bound wtIF2(GTP) was centered at an $\left\langle E_{\mathrm{FRET}}\right\rangle$ of $0.81 \pm 0.01$ that is outside the error of that observed for $30 \mathrm{~S} \mathrm{IC}_{\mathrm{wT}}$-bound wtIF2(GTP) ( $p$ value $=0.08$, Supplementary Table 1). This observation suggests that the separation between our labeling positions is $\sim 43 \AA$ in this subpopulation of $30 \mathrm{~S}_{\mathrm{IC}_{\mathrm{wT}}}$-bound wtIF2(GTP), a separation that is $\sim 3 \AA$ longer than what it is in a $30 \mathrm{~S}_{\mathrm{IC}_{\mathrm{wT}} \text {-bound wtIF2(GTP) }}$ that is active for rapid 50S subunit joining. The peak corresponding to the smaller, $44 \pm 12 \%$, subpopulation of $30 \mathrm{~S}$ $\mathrm{IC}_{\mathrm{wT} \text {,Met }}$-bound wtIF2(GTP) was centered at an even lower $\left\langle E_{\mathrm{FRET}}\right\rangle$ of $0.55 \pm 0.01$, indicating that the distance between our labeling positions is $\sim 53 \AA, \sim 13 \AA$ longer than what it is in $30 \mathrm{~S}$ $\mathrm{IC}_{\mathrm{wT}}$-bound wtIF2(GTP) that is active for rapid $50 \mathrm{~S}$ subunit joining. Even more dramatic results are obtained for the distribution of $E_{\mathrm{FRET}}$ values for the wIIF2(GTP)-bound state of $30 \mathrm{~S} \mathrm{IC}_{\mathrm{wT}, \mathrm{OH}}$ (Fig. $3 \mathrm{~b}$ and Supplementary Fig. $5 \mathrm{~g}$ ) in that the distribution exhibits only a single non-zero $E_{\mathrm{FRET}}$ peak that is centered at an $\left\langle E_{\mathrm{FRET}}\right\rangle$ of $0.53 \pm 0.02$ that is within error of that observed for the smaller subpopulation of $30 \mathrm{~S} \mathrm{IC}_{\mathrm{wT}, \mathrm{Met}}$-bound wtIF2(GTP) ( $p$ value $=0.4$, Supplementary Table 1$)$.

Our previous ensemble kinetic studies have shown that the rates of $50 \mathrm{~S}$ subunit joining to $30 \mathrm{~S}_{\mathrm{IC}_{\mathrm{wT}, \mathrm{Met}}}{ }^{*}$ and $30 \mathrm{~S}_{\mathrm{IC}_{\mathrm{wT}, \mathrm{OH}}}{ }^{*}$ are approximately fourfold and $\sim 15$-fold lower, respectively, than that to $30 \mathrm{~S} \mathrm{IC}_{\mathrm{wT}}{ }^{* 21}$. Given the values of $K_{\mathrm{d} \text {,wT,Met }}$ and $K_{\mathrm{d}, \mathrm{wT}, \mathrm{OH}}$ determined here and of the wtIF2 and $30 \mathrm{~S} \mathrm{IC}_{\mathrm{wT}, \text { Met }}{ }^{*}$ and $30 \mathrm{~S}$ $\mathrm{IC}_{\mathrm{wT}, \mathrm{OH}^{*}}{ }^{*}$ concentrations used in our previous kinetic studies ${ }^{21}$, we estimate that the occupancy of wtIF2(GTP) on $30 \mathrm{~S} \mathrm{IC}_{\mathrm{wT}, \mathrm{Met}}{ }^{*}$ and $30{\mathrm{~S} \mathrm{IC}_{\mathrm{wT}, \mathrm{OH}}}^{*}$ in our previous kinetic studies was approximately fivefold and $\sim 10$-fold lower, respectively, than that on $30 \mathrm{~S}$ $\mathrm{IC}_{\mathrm{wT}}{ }^{*}$ (Supplementary Table 2). It is notable that these estimated decreases in the occupancies of wtIF2(GTP) on $30 \mathrm{~S} \mathrm{IC}_{\mathrm{wT}, \text { Met }}{ }^{*}$ and $30 \mathrm{~S} \mathrm{IC}_{\mathrm{wT}, \mathrm{OH}^{*}}$ closely approximate the decreases in the rates of $50 \mathrm{~S}$ subunit joining to $30 \mathrm{~S} \mathrm{IC}_{\mathrm{wT}, \mathrm{Met}}{ }^{*}$ and $30 \mathrm{~S} \mathrm{IC}_{\mathrm{wT}, \mathrm{OH}}{ }^{*}$. Thus, it is possible that the lack of an $N$-formyl moiety or $N$-formylmethionine on $30 \mathrm{~S}$ IC-bound Met-tRNA ${ }^{\text {fMet }^{-}}$or tRNA ${ }^{\text {fMet }}$ decreases the rate of $50 \mathrm{~S}$ subunit joining by reducing the occupancy of wtIF2(GTP) on these pseudo 30S ICs to $19 \%$ and $\sim 9 \%$, respectively (Supplementary Table 2), rather than by stabilizing wtIF2(GTP) in an inactive conformation at an occupancy of nearly $100 \%$ on these pseudo 30 S ICs, as we have previously suggested ${ }^{21}$. The key question therefore becomes whether the activation of IF2(GTP) for rapid 50S subunit joining merely involves an fMet-tRNA ${ }^{\text {fMet }}$-dependent increase in the affinity of IF2(GTP) for the 30S IC or whether, in addition, there

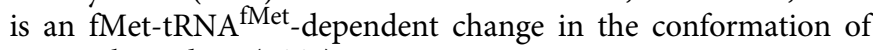
30S IC-bound IF2(GTP).

To address this question, we performed ensemble kinetic experiments to measure the rate of $50 \mathrm{~S}$ subunit joining to $30 \mathrm{~S}$ $\mathrm{IC}_{\mathrm{wT}, \mathrm{OH}}$ as a function of wtIF2(GTP) concentrations that were high enough to saturate $30 \mathrm{~S} \mathrm{IC}_{\mathrm{wT}, \mathrm{OH}}$ with wtIF2(GTP). As a
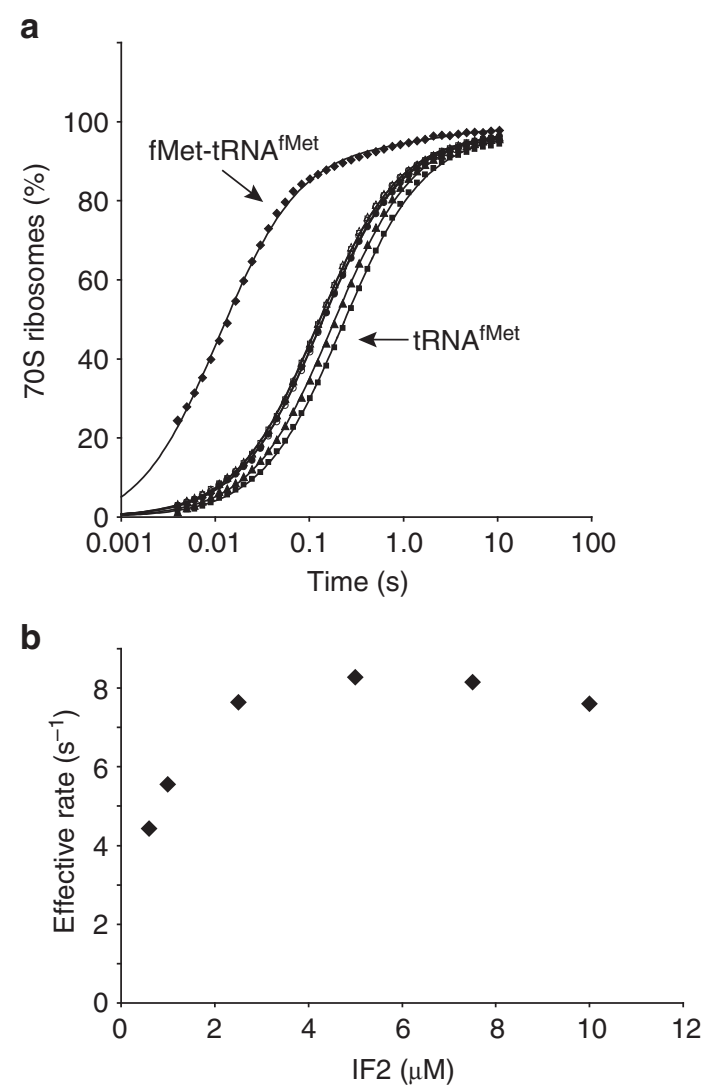

Fig. 4 Effect of IF2 concentration on the rate of 50 S subunit joining to a pseudo 30 S IC. a Ensemble kinetics of 70 S IC formation after rapid mixing of $50 \mathrm{~S}$ subunits with $30 \mathrm{~S} I \mathrm{C}_{\mathrm{wT}}$ assembled in the presence of $1 \mu \mathrm{M}$ wtIF2 or $30 \mathrm{~S} I \mathrm{C}_{\mathrm{WT}, \mathrm{OHS}}$ assembled in the presence of $0.6-10 \mu \mathrm{M}$ wtIF2. b Effective

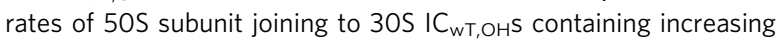
concentrations of wtIF2

reference, we measured the maximal rate of $50 \mathrm{~S}$ subunit joining to $30 \mathrm{~S}_{\mathrm{wT}}$ using a wtIF2(GTP) concentration of $1.0 \mu \mathrm{M}$ and obtained a rate of $\sim 80 \mathrm{~s}^{-1}$ (Fig. 4), a result that, in excellent agreement with our previous studies ${ }^{21}$, is $\sim 14$-fold faster than the rate of $50 \mathrm{~S}$ subunit joining to $30 \mathrm{~S} \mathrm{IC}_{\mathrm{wT}, \mathrm{OH}}$ measured at the same wtIF2(GTP) concentration. Titrating the concentration of wtIF2 (GTP) from 0.6 to $10 \mu \mathrm{M}$ using $30 \mathrm{~S} \mathrm{IC}_{\mathrm{wT}, \mathrm{OH}}$ resulted in a small, $\sim 1.5$-fold increase in the rate of $50 \mathrm{~S}$ subunit joining, suggesting that, at the $0.6 \mu \mathrm{M}$ concentrations of wtIF2(GTP) used in the previous studies, $30 \mathrm{~S} \mathrm{IC}_{\mathrm{wT}, \mathrm{OH}}$ was not saturated with wtIF2 (GTP). Nonetheless, we find that the rate of 50S subunit joining to $30 \mathrm{~S} \mathrm{IC}_{\mathrm{wT}, \mathrm{OH}}$ plateaus at a wtIF2(GTP) concentration of $\sim 2.5$ $\mu \mathrm{M}$, indicating that at wtIF2(GTP) concentrations above $\sim 2.5 \mu \mathrm{M}$, $30 \mathrm{~S} \mathrm{IC}_{\mathrm{wT}, \mathrm{OH}}$ is saturated with wtIF2(GTP). Interestingly, we find that, even when $30 \mathrm{~S} \mathrm{IC}_{\mathrm{wT}, \mathrm{OH}}$ is saturated with wtIF2(GTP), the rate of $50 \mathrm{~S}$ subunit joining is still $\sim 11$-fold lower than the maximal rate of $50 \mathrm{~S}$ subunit joining to $30 \mathrm{~S} \mathrm{IC}_{\mathrm{wT}}$. Based on these results, we conclude that the decreased rate of $50 \mathrm{~S}$ subunit joining originates from a conformation of $30 \mathrm{~S} \mathrm{IC}_{\mathrm{wT}, \mathrm{OH}^{-}}$bound wtIF2 (GTP) that is inactive for rapid 50S subunit joining.

The conformation of dI-dIII confers rapid subunit joining. To investigate whether and how the activating mutation in dIII modulates the affinity of IF2(GTP) for pseudo 30S ICs and the conformation of the resulting pseudo 30S IC-bound IF2(GTP), we performed smFRET experiments using mutIF2[Cy5 $]_{\mathrm{dIV}}(\mathrm{GTP})$ and $30 \mathrm{~S} \mathrm{IC}_{\mathrm{mT}, \mathrm{Met}}$ or $30 \mathrm{~S} \mathrm{IC}_{\mathrm{mT}, \mathrm{OH}}$. The results of these experiments demonstrate that excursions to the mutIF2(GTP)-bound 


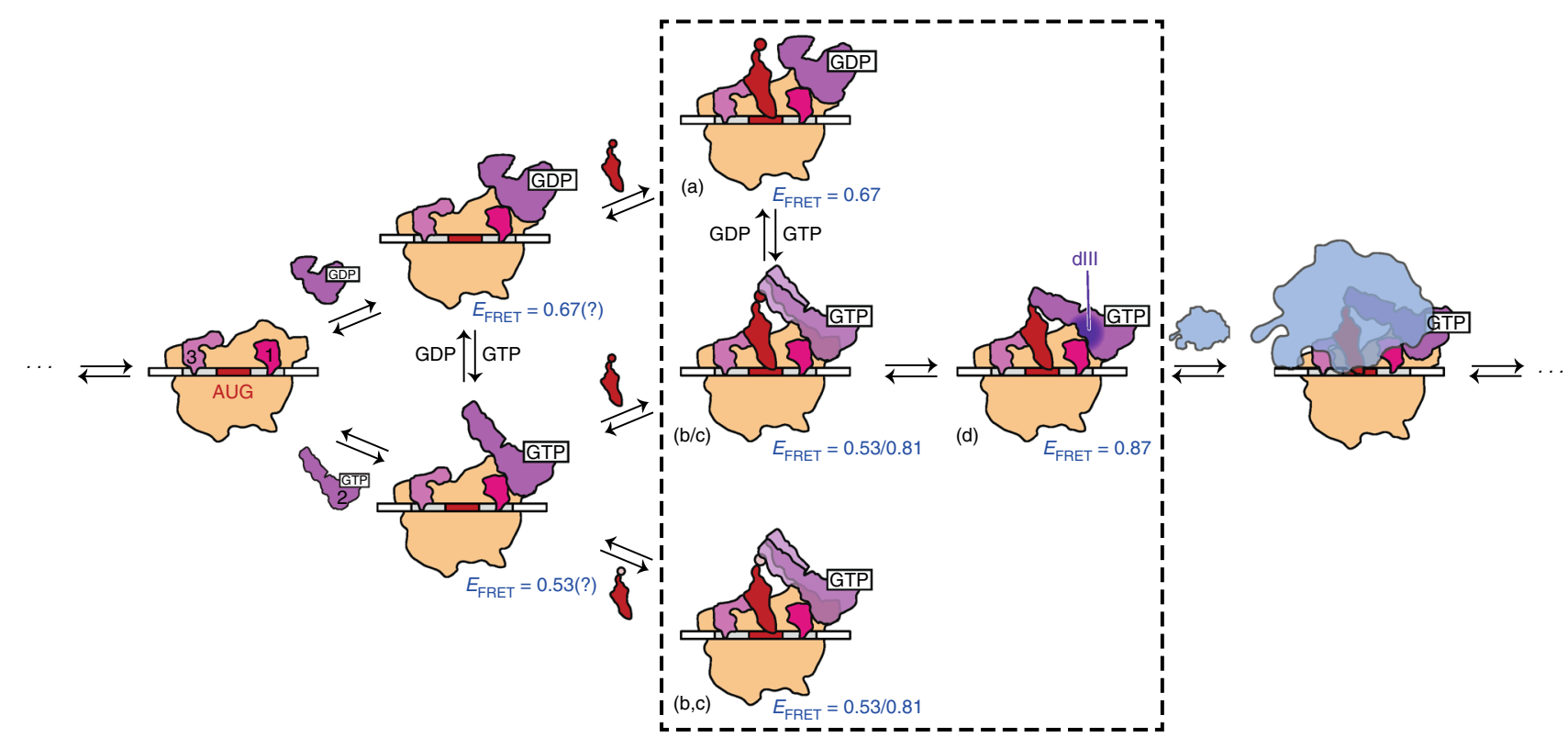

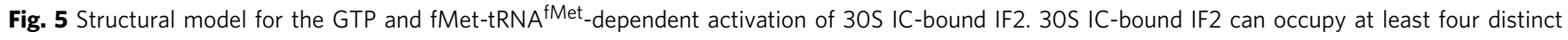
conformational states relative to the P-site tRNA (denoted as conformations $a-d$ ). These conformational states are characterized by $E_{\mathrm{FRET}}$ values of 0.67 $(a), 0.53(b), 0.81(c)$, and $0.87(d)$. The dotted box highlights $30 \mathrm{~S} \mathrm{ICs}$ and pseudo $30 \mathrm{~S} \mathrm{ICS} \mathrm{studied} \mathrm{in} \mathrm{this} \mathrm{work} \mathrm{and} \mathrm{their} \mathrm{corresponding} E_{\mathrm{FRET}}$ values. $30 \mathrm{~S}$ ICs and $E_{\text {FRET }}$ values indicated outside of the dotted box are predicted conformational states of IF2. (Central panel) The specific binding of GTP to dl of IF2 is allosterically communicated through dIII and results in a repositioning of dIV closer to the P site of the $30 \mathrm{~S} \mathrm{IC}$ and further from dI-III. The specific recognition of the $\mathrm{N}$-formyl-methionine of a P-site-bound fMet-tRNA ${ }^{\text {fMet }}$ by dIV of IF2(GTP) feeds back to dIII (dark purple), thereby stabilizing a conformation of dl-dlll of IF2 that is active for rapid 50S subunit joining. In contrast, (top panel) the binding of GDP to dl of IF2, or (bottom panel) the presence of an unformylated Met-tRNA ${ }^{\mathrm{fMet}}$, or an elongator tRNA in the P site fails to stabilize the active conformation of IF2, instead leaving IF2 in a conformation(s) that are inactive for rapid 505 subunit joining

state in the $30 \mathrm{~S} \mathrm{IC}_{\mathrm{mT}, \mathrm{Met}}$ and $30 \mathrm{~S} \mathrm{IC}_{\mathrm{mT}, \mathrm{OH}} E_{\mathrm{FRET}}$ trajectories are significantly longer than those to the wtIF2(GTP)-bound states in the $30 \mathrm{~S} \mathrm{IC}_{\mathrm{wT}, \mathrm{Met}}$ and $30 \mathrm{~S} \mathrm{IC}_{\mathrm{wT}, \mathrm{OH}} E_{\mathrm{FRET}}$ trajectories (compare Fig. $3 c$, d with Fig. $3 \mathrm{a}, \mathrm{b}$ ). Consistent with this, we find that $K_{\mathrm{d}, \mathrm{mT}}$, Met and $K_{\mathrm{d}, \mathrm{mT}, \mathrm{OH}}$ are $\sim 20$-fold and $\sim 30$-fold smaller than $K_{\mathrm{d}, \mathrm{wT}, \text { Met }}$ and $K_{\mathrm{d}, \mathrm{wT}, \mathrm{OH}}$, respectively (Table 1 ). The activating mutation in dIII therefore enables mutIF2(GTP) to bind to $30 \mathrm{~S}_{\mathrm{IC}_{\mathrm{mT}, \mathrm{Met}} \text { and }}$ $30 \mathrm{SC}_{\mathrm{mT}, \mathrm{OH}}$ with an affinity that is over an order of magnitude higher than that with which wtIF2(GTP) binds to $30 \mathrm{~S} \mathrm{IC}_{\mathrm{wT}, \text { Met }}$ and $30 \mathrm{~S} \mathrm{IC}_{\mathrm{wT}, \mathrm{OH}}$. This demonstrates that high-affinity binding of IF2(GTP) to the 30S IC does not necessarily require dIV to establish direct interactions with the $N$-formyl moiety or $N$-formyl-methionine of the P site-bound fMet-tRNA ${ }^{\text {fMet }}$. Rather, it is the conformation of dI-dIII that contributes significantly to the affinity of IF2(GTP) for the 30S IC. Such a contribution could arise from direct interactions between $\mathrm{dIII}$ and $\mathrm{S} 12$ or some other component of the 30 S IC and/or from allosteric modulation of the interactions that dII makes with h5 and h14 of 16s rRNA.

Interestingly, the distribution of $E_{\mathrm{FRET}}$ values for the mutIF2 (GTP)-bound state of $30 \mathrm{~S} \mathrm{IC}_{\mathrm{mT}, \mathrm{Met}}$ and $30 \mathrm{~S} \mathrm{IC}_{\mathrm{mT}, \mathrm{OH}}$ are very similar to those for the wtIF2(GTP)-bound state of $30 \mathrm{~S} \mathrm{IC}_{\mathrm{wT}, \text { Met }}$ and $30 \mathrm{~S} \mathrm{IC}_{\mathrm{wT}, \mathrm{OH}}$. Specifically, the distribution of $E_{\mathrm{FRET}}$ values for the mutIF2(GTP)-bound state of $30 \mathrm{~S} \mathrm{IC}_{\mathrm{mT}, \mathrm{Met}}$ (Fig. $3 \mathrm{c}$ and Supplementary Fig. 5f) exhibited two non-zero $E_{\text {FRET }}$ peaks. The first peak encompassed a smaller, $42 \pm 5.7 \%$, subpopulation of the mutIF2(GTP)-bound state of $30 \mathrm{~S}_{\mathrm{mT}, \mathrm{Met}}$ and was centered at an $\left\langle E_{\text {FRET }}>\right.$ of $0.83 \pm 0.04$ that is within error of that observed for the larger subpopulation of the wtIF2(GTP)-bound state of $30 \mathrm{~S}$ $\mathrm{IC}_{\mathrm{wT}, \text { Met }}(p$ value $=0.7$, Supplementary Table 1$)$. The second peak encompassed a larger, $58 \pm 5.7 \%$, subpopulation of the mutIF2 (GTP)-bound state of $30 \mathrm{~S} \mathrm{IC}_{\mathrm{mT}, \mathrm{Met}}$ and was centered at an $<E_{\mathrm{FRET}}>$ of $0.57 \pm 0.02$ that is also within error of that observed for the smaller subpopulation of the wtIF2(GTP)-bound state of $30 \mathrm{~S}_{\mathrm{wT}, \mathrm{Met}}(p$ value $=0.4$, Supplementary Table 1$)$. Similarly, the distribution of $E_{\mathrm{FRET}}$ values for the mutIF2(GTP)-bound state of $30 \mathrm{~S} \mathrm{IC}_{\mathrm{mT}, \mathrm{OH}}$ (Fig. $3 \mathrm{~d}$ and Supplementary Fig. 5h) exhibited a single non-zero $E_{\text {FRET }}$ peak centered at an $\left\langle E_{\text {FRET }}>\right.$ of $0.57 \pm 0.01$ that is within error of that observed for the larger subpopulation of the mutIF2(GTP)-bound state $30 \mathrm{~S} \mathrm{IC}_{\mathrm{mT}, \mathrm{Met}}$ and the wtIF2 (GTP)-bound state of $30 \mathrm{~S} \mathrm{IC}_{\mathrm{wT}, \mathrm{OH}}$ ( $p$ value $=0.8$, Supplementary Table 1). The fact that the $\left\langle E_{\mathrm{FRET}}\right\rangle \mathrm{s}$ that we observe for $30 \mathrm{~S}$ $\mathrm{IC}_{\mathrm{mT}, \mathrm{Met}^{-}}$and 30S $\mathrm{IC}_{\mathrm{mT}, \mathrm{OH}^{-}}$-bound mutIF2(GTP), here are within error of the $\left\langle E_{\mathrm{FRET}}>\mathrm{s}\right.$ observed for $30 \mathrm{~S} \mathrm{IC}_{\mathrm{wT}, \mathrm{Met}^{-}}$and $30 \mathrm{~S} \mathrm{IC}_{\mathrm{wT}}$, $\mathrm{OH}^{- \text {bound }}$ wtIF2(GTP) strongly suggests that the activating mutation in dIII does not significantly alter the positions of dIV of mutIF2(GTP) in $30 \mathrm{~S} \mathrm{IC}_{\mathrm{mT}, \mathrm{Met}}$ and $30 \mathrm{~S} \mathrm{IC}_{\mathrm{mT}, \mathrm{OH}}$ relative to those of dIV of wtIF2(GTP) in $30 \mathrm{SC}_{\mathrm{wT}, \text { Met }}$ and $30 \mathrm{~S} \mathrm{IC}_{\mathrm{wT}, \mathrm{OH}}$. Indeed, with the exception of a relatively small, $\sim 15 \%$, shift in the subpopulation occupancies of the IF2(GTP)-bound states of $30 \mathrm{~S}$ $\mathrm{IC}_{\mathrm{wT}, \text { Met }}$ and 30S IC $\mathrm{mT}_{\mathrm{m}, \text { Met }}$, dIV of wtIF2(GTP) and mutIF2(GTP) seem to adopt similar conformations in 30S ICs carrying MettRNA ${ }^{\text {fMet }}$ and tRNA ${ }^{\text {fMet }}$.

Previously, we have used ensemble kinetic experiments to show that the rates of $50 \mathrm{~S}$ subunit joining to $30{\mathrm{~S} \mathrm{IC}_{\mathrm{mT}, \mathrm{Met}}}^{*}$ and $30 \mathrm{~S}$ $\mathrm{IC}_{\mathrm{mT}, \mathrm{OH}^{*}}$ are approximately fourfold and $\sim 12$-fold higher than to $30 \mathrm{~S} \mathrm{IC}_{\mathrm{WT}, \mathrm{Met}}{ }^{*}$ and $30 \mathrm{~S} \mathrm{IC}_{\mathrm{WT}, \mathrm{OH}^{*} \text {, respectively }}{ }^{21}$. Thus, the activating mutation in dIII enables mutIF2(GTP) to catalyze $50 \mathrm{~S}$ subunit joining to $30 \mathrm{~S} \mathrm{IC}_{\mathrm{mT}, \mathrm{Met}}{ }^{*}$ and $30 \mathrm{~S} \mathrm{IC}_{\mathrm{mT}, \mathrm{OH}}{ }^{*}$ at rates that are within $30 \%$ of those observed for $30 \mathrm{~S}_{\mathrm{IC}_{\mathrm{wT}}}^{*}$ and $30 \mathrm{~S}$ $\mathrm{IC}_{\mathrm{mT}}{ }^{\star}$. Based on the results reported here, we propose that the activating mutation in dIII enables this increase in the rate of subunit joining by stabilizing a conformation of dI-dIII, that not only increases the affinity of mutIF2(GTP) for $30 \mathrm{~S} \mathrm{IC}_{\mathrm{mT}, \mathrm{Met}}$ and $30 \mathrm{SC}_{\mathrm{mT}, \mathrm{OH}}$, but that is optimized for the rapid recruitment ${ }^{35,36}$ 
and/or docking of the $50 \mathrm{~S}$ subunit onto $30 \mathrm{~S}_{\mathrm{IC}} \mathrm{m}_{\text {,Met }}$ and $30 \mathrm{~S}$ $\mathrm{IC}_{\mathrm{mT}, \mathrm{OH}}$. We speculate that, in the context of wtIF2(GTP), this conformation of dI-dIII is rendered conditional on the direct interactions of dIV with the $\mathrm{N}$-formyl-methionine.

\section{Discussion}

Here, we have used a combination of smFRET and ensemble kinetic studies of $50 \mathrm{~S}$ subunit joining to elucidate the mechanism of IF2 activation for rapid 50S subunit joining to the 30S IC. Our results demonstrate how GTP and fMet-tRNA ${ }^{\text {fMet }}$ stabilize the specific conformation of 30S IC-bound IF2 that confers highaffinity binding to the 30S IC and rapid 50S subunit joining. Based on our findings, we propose a model for IF2 activation during translation initiation (Fig. 5). In this model, four conformations of 30S IC-bound IF2, which we denote as conformations $a-d$ in Fig. 5, play major roles. In conformation $a$, dI is not in the GTP-bound form, dII and dIII do not interact fully with the $30 \mathrm{~S}$ subunit, and dIV is positioned closer to dI-dIII than to the P site-bound tRNA. This conformation corresponds to the $\left\langle E_{\mathrm{FRET}}>\right.$ of $0.67 \pm 0.01$ that we observe for $30 \mathrm{~S} \mathrm{IC}_{\mathrm{wD}}$-bound wtIF2(GDP) and it is inactive for rapid 50S subunit joining. In conformation $b, \mathrm{dI}$ is in the GTP-bound form, dII and dIII have established increased interactions with the 30S subunit, and dIV is positioned closer to the P site-bound tRNA than to dI-dIII. This GTP-dependent repositioning of dIV is driven by an allosteric mechanism in which binding of GTP to dI triggers a conformational change and/or repositioning of dI-dIII, which ultimately places dIV closer to the P site. Independent evidence in favor of our model comes from structural studies in which binding of GTP to dI results in a restructuring of dI-dIII that has been predicted to modulate the interactions of dII with h14 and $\mathrm{h} 5$ of $16 \mathrm{~s} \mathrm{rRNA}^{37}$ and of dIII with $\mathrm{S} 12^{27}$. In conformation $b$, which corresponds to the $\left\langle E_{\mathrm{FRET}}\right\rangle$ value of $0.53 \pm 0.02$ that we observe for $30 \mathrm{SIC}_{\mathrm{wT}, \mathrm{OH}}$-bound wtIF2(GTP), dIV does not make any stabilizing contacts with fMet-tRNA ${ }^{\text {fMet }}$. Conformation $c$, which corresponds to the $\left\langle E_{\mathrm{FRET}}\right\rangle$ of $0.81 \pm 0.01$ that we observe in $30 \mathrm{~S} \mathrm{IC}_{\mathrm{wT}, \text { Met }}$-bound wtIF2(GTP), is similar to the second conformation, except that dIV now makes partial interactions with the $\mathrm{N}$-formyl-methionine of a P-site fMet-tRNA ${ }^{\mathrm{fMet}}$. In conformation $d$, which corresponds to the $\left\langle E_{\mathrm{FRET}}\right\rangle$ of $0.87 \pm 0.02$ that we observe in $30 \mathrm{~S} \mathrm{IC}_{\mathrm{wT}}$-bound wtIF2(GTP), dIV interacts fully with the $N$-formyl-methionine of the P-site fMet-tRNA ${ }^{\text {fMet }}$ and has adopted a position that allosterically feeds back and stabilizes the conformation of dI-dIII that is active for rapid 50S subunit joining. Independent evidence in support of the idea that the interactions between $\mathrm{dIV}$ and the $\mathrm{N}$-formyl-methionine allosterically feed back to dI-dIII in order to stabilize a conformation that is active for rapid 50S subunit joining comes from previous measurements of the affinity of dI for GTP in which it was observed that the presence of a P-site fMet-tRNA ${ }^{\mathrm{fMet}}$ allosterically increases the affinity of dI for GTP ${ }^{38}$.

A major finding of this study is that dIII integrates GTP binding to $\mathrm{dI}$ and fMet-tRNA ${ }^{\mathrm{fMet}}$ recognition by dIV in order to allosterically regulate the affinity of IF2 for the 30S IC and the conformation of the resulting 30S IC-bound IF2. This suggests that dIII may serve as a target for antibiotics that inhibit IF2 allosterically. To explore this option, further characterization of the mechanism of IF2 activation and, in particular, the dynamic interplay between dI-dIV will be necessary. This will require the development of additional, intramolecular labeling schemes to directly probe the interdomain dynamics of IF2. In addition, while low- to high-resolution cryo-EM structures of the active conformation of 30 S IC-bound IF2 have been reported ${ }^{16-18}$, a comprehensive structural understanding of IF2 activation will undoubtedly require not only higher resolution cryo-EM or crystallographic structures of the active conformation of 30S ICbound IF2, but also the inactive conformation(s) of 30S IC-bound IF2 (e.g., $30 \mathrm{~S} \mathrm{IC}_{\mathrm{wD}}$, 30S $\mathrm{IC}_{\mathrm{wT}, \mathrm{Met}}$, or $\left.30 \mathrm{~S} \mathrm{IC}_{\mathrm{wT}, \mathrm{OH}}\right)$.

\section{Methods}

Preparation and fluorescence labeling of IFs. pProEX-HTb expression vectors containing a cloned copy of the E. coli IF1 gene or a cloned copy of the E. coli IF2 gene ( $\gamma$-isoform) downstream of a six-histidine (6xHis) tag were transformed into the BL21(DE3) strain and purified as described previously ${ }^{28,39}$. Briefly, transformed bacteria was grown in Terrific Broth at $37^{\circ} \mathrm{C}$ until the bacterial cell culture reached an optical density $\left(\mathrm{OD}_{600}\right)$ of $\sim 0.8$. Overexpression of the IFs was induced by the addition of $1 \mathrm{mM}$ Isopropyl $\beta$-D-1-thiogalactopyranoside and the induced cultures were grown for an additional $2-4 \mathrm{~h}$ at $37^{\circ} \mathrm{C}$. 6xHis-tagged IF1 and IF2 were purified by $\mathrm{Ni}^{2+}$-nitrilotriacetic acid affinity chromatography using the batch procedure (Qiagen). Subsequently, the N-terminal 6xHis tags were removed by incubating the purified IFs with the tobacco etch virus protease. Further purification of untagged IF1 was achieved using a HiLoad 16/60 Superdex 75 prep grade gel filtration column and further purification of untagged IF2 was achieved using a HiTrap SP HP cation exchange column. In both instances, peak fractions were collected, concentrated using an Amicon centrifugal filtration device, and the IFs were buffer exchanged into $2 \times$ IF storage buffer $\left(20 \mathrm{mM}\right.$ Tris-OAc $\left(\mathrm{pH}_{\mathrm{RT}}=6.9\right)$, $100 \mathrm{mM} \mathrm{KCl}, 20 \mathrm{mM} \mathrm{Mg}(\mathrm{OAc})_{2}$, and $\left.10 \mathrm{mM} \beta \mathrm{ME}\right)$. Following the addition of 1 volume of $100 \%$ glycerol, the purified IFs were stored at $-20^{\circ} \mathrm{C}$. The concentration of IF1 was measured using the Bradford assay, and the concentration of IF2 was determined by measuring its ultraviolet absorbance at $280 \mathrm{~nm}$ and by using its molar extinction coefficient $\left(27390 \mathrm{M}^{-1} \mathrm{~cm}^{-1}\right)$, which was calculated using the ProtParam tool on the ExPASy Proteomics Server.

Mutant variants of IF2 harboring either (1) a serine-to-tyrosine mutation at amino acid 753 (IF2(Ser753Tyr)), (2) a glycine-to-cysteine mutation at amino acid position 810 (IF2(Gly810Cys)), (3) a Gly810Cys and a Ser753Tyr mutation (IF2 (S753Y-G810C)), or (4) an alanine-to-cysteine mutation at amino acid position 760 (IF2(Ala760Cys)) were generated using the mutagenic DNA primers shown below and the PfuUltra High-Fidelity DNA Polymerase (Agilent Technologies) following the manufacturer's protocol.

Gly810Cys:

5'-CGCCGAAATTTTGTGCCATCGCAGGCTGTATG-3'

5'-CATACAGCCTGCGATGGCACAAAATTTCGGCG-3'

Ser753Tyr:

5'-GCTTTAACGTACGTGCTGATGCCTAT-3'

5'-CCGCTTCAATCACTTTACGTGCATAG-3'.

Ala760Cys:

5'-CTCTGCACGTAAAGTGATTGAATGCGAA-3'

5'-CGCAGATCCAGGCTTTCGCATTCAATCAC-3'.

The mutant IF2 constructs were verified by automated DNA sequencing. The overexpression and purification of these IF2 variants was performed exactly as described above for wild-type IF2.

The cysteine residue introduced into amino acid position 810 (cysteine-810) in the IF2(Gly810Cys) and IF2(Ser753Tyr-Gly810Cys) variants or into amino acid position 760 (cysteine-760) in the IF2(Ala760Cys) variant was used to label IF2 with the maleimide-derivatized Cyanine 5, or Cy5, fluorophore as described previously ${ }^{11,28}$ and generate wtIF $2[\mathrm{Cy} 5]_{\mathrm{dIV}}$ and mutIF2 [Cy5 $]_{\mathrm{dIV}}$ or wtIF $2[\mathrm{Cy} 5]_{\mathrm{dIII}}$, respectively. Briefly, IF2(Gly810Cys), IF2(Ser753Tyr-Gly810Cys), and IF2

(Ala760Cys) were buffer exchanged into labeling buffer containing a 10-fold molar excess of tris(2-carboxyethyl)phosphine hydrochloride using a Micro Bio-Spin P6 gel filtration spin column and incubated at room temperature for $30 \mathrm{~min}$. To specifically and quantitatively label IF2 at either cysteine-810 and cysteine-760, a 10 -fold molar excess of Cy5 dissolved in anhydrous dimethylsulfoxide was added to the labeling reaction and incubated overnight at $4{ }^{\circ} \mathrm{C}$. Cy5-labeled IF2 was separated from free, unreacted Cy 5 using gel filtration chromatography. The labeling efficiency of IF2 at cysteine-810 ( 90\%) and IF2 at cysteine-760 ( 80\%) was determined from the ratio of the concentration of IF2 and the concentration of Cy5. The concentration of Cy5 was determined by measuring its absorbance at $650 \mathrm{~nm}$ and using its molar extinction coefficient $\left(250,000 \mathrm{M}^{-1} \mathrm{~cm}^{-1}\right)$. The concentration of IF2 was corrected for the $5 \%$ absorbance of Cy5 at $280 \mathrm{~nm}$.

Preparation of 30 S subunits. Highly active $30 \mathrm{~S}$ subunits were purified from the E. coli strain MRE600 as described previously ${ }^{28}$. Briefly, clarified cell lysates were centrifuged through a sucrose cushion solution $\left(20 \mathrm{mM}\right.$ Tris- $\mathrm{HCl}\left(\mathrm{pH}_{4}{ }^{\circ} \mathrm{C}=7.2\right)$, $500 \mathrm{mM} \mathrm{NH} 4 \mathrm{Cl}, 10 \mathrm{mM} \mathrm{MgCl} 2,0.5 \mathrm{mM}$ EDTA, $6 \mathrm{mM} \beta \mathrm{ME}, 37.7 \%$ sucrose) to isolate crude ribosomes. Next, crude ribosomes were centrifuged through a $10-40 \%$ sucrose density gradient to isolate tight-coupled $70 \mathrm{~S}$ ribosomes. To promote the dissociation of tight-coupled $70 \mathrm{~S}$ ribosomes into $30 \mathrm{~S}$ and $50 \mathrm{~S}$ ribosomal subunits, the tight-coupled $70 \mathrm{~S}$ ribosomes were dialyzed into ribosome dissociation buffer (10 mM Tris-OAc $\left(\mathrm{pH}_{4}{ }^{\circ} \mathrm{C}=7.5\right), 60 \mathrm{mM} \mathrm{NH}_{4} \mathrm{Cl}, 1 \mathrm{mM} \mathrm{MgCl}_{2}, 0.5 \mathrm{mM}$ EDTA, $6 \mathrm{mM} \beta \mathrm{ME}$ ). To isolate $30 \mathrm{~S}$ subunits, the dissociated ribosomes were centrifuged through a $10-40 \%$ sucrose gradient prepared in ribosome dissociation buffer. Purified 30S subunits were pelleted by ultra-centrifugation, re-suspended in ribosome storage buffer $\left(10 \mathrm{mM}\right.$ Tris-OAc $\left(\mathrm{pH}_{4}{ }^{\circ} \mathrm{C}=7.5\right), 60 \mathrm{mM} \mathrm{NH}_{4} \mathrm{Cl}, 7.5 \mathrm{mM}$ 
$\mathrm{MgCl}_{2}, 0.5 \mathrm{mM}$ EDTA, $\left.6 \mathrm{mM} \beta \mathrm{ME}\right)$, quantified by measuring the ultraviolet absorbance at $260 \mathrm{~nm}\left(1 \mathrm{~A}_{260}\right.$ unit $\left.=79 \mathrm{nM}\right)$, and stored in small aliquots at $-80^{\circ} \mathrm{C}$.

Preparation of mRNAs. The 5'-biotinylated mRNA used in our single molecule experiments (5'-bio-mRNA $\mathrm{AUG}_{\mathrm{AUG}}$ ) and the mRNA used in our primer-extension inhibition, or "toeprinting"-based IF2 activity assay (mRNApri-ext) were both variants of the mRNA encoding gene product 32 from T4 bacteriophage. $5^{\prime}$-bio$\mathrm{mRNA}_{\mathrm{AUG}}$ was chemically synthesized and mRNApri-ext was generated by in vitro transcription using T7 RNA polymerase as previously described ${ }^{28}$. The mRNA sequence for 5'-bio-mRNA $\mathrm{AUG}_{\text {and }} \mathrm{mRNApri-ext}$ are shown below. In both of the mRNA sequences, the Shine-Dalgarno (SD) sequence is underlined, the start codon is underlined and bolded, and the spacer sequence between the SD sequence and the start codon is italicized. In addition, for mRNApri-ext, the primer annealing site used to reverse transcribe the message is underlined, bolded, and italicized. The mMFTI mRNA used in our light-scattering experiments is also shown below and the SD sequence, start codon, and spacer sequence are highlighted as indicated for 5'-bio-mRNA ${ }_{\mathrm{AUG}}$ and mRNApri-ext.

5'-bio-mRNA ${ }_{\text {AUG }}$ :

5'-biotin-CAACCUAAAACUUACACAAAUUAAAAAGGAAAUAGACAUG UUCAAAGUCGAAAAAUCUACUGCU-3'.

mRNApri-ext:

5'-GGCAACCUAAAACUUACACAGGGCCCUAAGGAAAUAAAAAUG UUUAAAGAAGUAUACAC UGCUGAACUCGCUGCACA

AAUGGCUAAACUGAAUGGCAAUAAAGGUUUUUCUUCU

GAAGAUAAAGGCGAGUGGAAACUGAAACUCGAUAA

UGCGGGUAACGGUCAAGCAGUAAUUCGUUUUCUU

CCGUCUAAAAAUGAUGAACAAGCACCAUUCGCAAUUC

UUGUAAAUCACGGUUUCAAGAAAAAUGGUAAAUGGUA

UAUUGAAACAUGUUCAUCUACCCAUGGUGAUUACGAU

UCUUGCCCAGUAUGUCAAUACAUCAGUAAAAAUGAU

CUAUACAACACUGACAAUAAAGAGUACAGUCUUGU

UAAACGUAAAACUUCUUACUGGGCUAACAUUCUUGU

AGUAAAAGACCCAGCUGCUCCAGAAAACGAAGGUAA

AGUAUUUAAAUACCGUUUCGGUAAGAAAAUCUGGG

AUAAAAUCAAUGCAAUGAUUGCGGUUGAUGUUGAA

AUGGGUGAAACUCCAGUUGAUGUAACUUGUCCGUG

GGAAGGUGCUAACUUUGUACUGAAAGUUAAACAAG

UUUCUGGAUUUAGUAACUACGAUGAAUCUAAAUUCCU

GAAUCAAUCUGCGAUUCCAAACAUUGACGAUGAAUCU

UUCCAGAAAGAACUGUUCGAACAAAUGGUCGACCUU

UCUGAAAUGACUUCUAAAGAUAAAUAAGG-3'.

mMFTI MRNA:

5'-GGGAAUUCGGGCCCUUGUUAACAAUUAAGGAGGUAUACUAUG UUUACGAUUUAAUUGCAGAAAAAAAAAAAAAAAAAAAAA-3'.

Preparation and fluorescence labeling of tRNAs. $\mathrm{RNAA}^{\mathrm{fMet}}$ was aminoacylated and formylated using purified methionyl-tRNA synthetase and methionyl-tRNA formyltransferase following previously published protocols ${ }^{39}$. The aminoacylation and formylation efficiency was $>90 \%$ as determined after separating the tRNA ${ }^{\mathrm{fMet}}$, Met-tRNA $^{\mathrm{fMet}}$, and fMet-tRNA ${ }^{\mathrm{fMet}}$ species using a TSKgel Phenyl-5PW hydrophobic interaction chromatography (HIC) column. tRNA ${ }^{\text {fMet }}$ was labeled by reacting its naturally occurring 4 -thiouridine at nucleotide position 8 with a maleimide-derivatized cyanine 3 , or $\mathrm{Cy} 3$, as described previously ${ }^{28}$. Cy3-labeled tRNA $^{\text {fMet }}$ was separated from free, unreacted Cy3 by extensive phenol extraction, and from unlabeled tRNA ${ }^{\text {fMet }}$ using HIC chromatography.

Preparation of 30 ICs. For smFRET experiments, 30S ICs were assembled by combining $0.6 \mu \mathrm{M}$ Cy3-labeled initiator tRNA (fMet-tRNA(Cy3) fMet $^{\text {, Met-tRNA }}$ $(\mathrm{Cy} 3)^{\mathrm{fMet}}$, or tRNA(Cy3) $\left.{ }^{\mathrm{fMet}}\right), 0.9 \mu \mathrm{M}$ IF1, $0.9 \mu \mathrm{M}$ Cy5-labeled wildtype or mutant IF2 (wtIF2[Cy5 $]_{\mathrm{dIV}}$, mutIF2[Cy5] $]_{\mathrm{dIV}}$, or wtIF2[Cy5 $\left.]_{\mathrm{dIII}}\right), 1.8 \mu \mathrm{M} 5^{\prime}$-biotinylated mRNA and $0.6 \mu \mathrm{M}$ purified E. coli $30 \mathrm{~S}$ subunits in Tris-polymix buffer $(50 \mathrm{mM}$ Tris-OAc ( $\mathrm{pH}_{\mathrm{RT}}$ of 7.5), $100 \mathrm{mM} \mathrm{KCL}, 5 \mathrm{mM} \mathrm{NH}_{4} \mathrm{OAc}, 5 \mathrm{mM} \mathrm{Mg}(\mathrm{OAc})_{2}, 0.1 \mathrm{mM}$ EDTA, $1 \mathrm{mM}$ GTP (or $1 \mathrm{mM} \mathrm{GDP}$ ), $5 \mathrm{mM}$ putrescine- $\mathrm{HCl}, 1 \mathrm{mM}$ spermidine-free base, and $6 \mathrm{mM} \beta \mathrm{ME}$. Reactions were incubated at $37^{\circ} \mathrm{C}$ for $10 \mathrm{~min}$ and transferred to ice for an additional $5 \mathrm{~min}$. Small aliquots were prepared, flash-frozen with liquid nitrogen, and stored at $-80^{\circ} \mathrm{C}$.

For the toeprinting experiments, $30 \mathrm{~S}$ ICs were assembled in a Tris-Polymix buffer containing $3 \mathrm{mM} \mathrm{Mg}(\mathrm{OAc})_{2}$ as described previously 28,39 . Briefly, $0.5 \mu \mathrm{M} 30 \mathrm{~S}$ subunits, $5 \mu \mathrm{M}$ of the indicated IF2 variant, and $0.8 \mathrm{mM}$ GTP were combined and incubated for $10 \mathrm{~min}$ at $37^{\circ} \mathrm{C}$. Next, $0.25 \mu \mathrm{M}$ of mRNApri-ext pre-annealed with a $5^{\prime}\left[{ }^{32} \mathrm{P}\right]$-labeled DNA primer of sequence TATTGCCATTCAGTTTAG was added to the reaction and incubated for an additional $10 \mathrm{~min}$ at $37^{\circ} \mathrm{C}$. The DNA primer was radioactively labeled with $\left[\gamma^{-32} \mathrm{P}\right] \mathrm{ATP}$ and T4 polynucleotide kinase (New England Biolabs) using the manufacturer's protocol. Finally, $0.8 \mu \mathrm{M}$ fMet$\mathrm{tRNA}^{\mathrm{fMet}}$, and/or tRNA ${ }^{\mathrm{Phe}}$ was added to the reaction and incubated for an additional $10 \mathrm{~min}$ at $37^{\circ} \mathrm{C}$.

For light-scattering experiments, 30S ICs were assembled in $4^{\prime}$ (2-hydroxyethyl)1-piperazineethanesulfonic acid (HEPES)-Polymix buffer (30 mM HEPES $\mathrm{pH}_{\mathrm{RT}=7.5}, 95 \mathrm{mM} \mathrm{KCl}, 5 \mathrm{mM} \mathrm{NH} \mathrm{H}_{4} \mathrm{Cl}, 0.5 \mathrm{mM}$ Calcium chloride $\left(\mathrm{CaCl}_{2}\right), 8 \mathrm{mM}$ putrescine- $\mathrm{HCl}, 1 \mathrm{mM}$ spermidine free base, $6 \mathrm{mM} \mathrm{Mg}(\mathrm{OAc})_{2}, 2 \mathrm{mM}$ phosphoenolpyruvate, $1 \mathrm{mM} \mathrm{GTP}, 1 \mathrm{mM} \mathrm{ATP}, 1 \mu \mathrm{g} \mathrm{ml}^{-1}$, pyruvate kinase, and 1 $\mu \mathrm{g} \mathrm{ml}^{-1}$ of myokinase) as described previously ${ }^{21}$. Briefly, $0.32 \mu \mathrm{M} 30 \mathrm{~S}$ subunits were combined with $0.8 \mu \mathrm{M}$ mMFTI mRNA, $1 \mu \mathrm{M}$ IF1, either $0.6 \mu \mathrm{M}$ IF2 (Supplementary Fig. 3), or 0.6-10 $\mu \mathrm{M}$ IF2 (Fig. 4), and either $0.9 \mu \mathrm{M}$ fMettRNA $^{\mathrm{fMet}}$, or $1.6 \mu \mathrm{M}$ deacylated tRNA ${ }^{\mathrm{fMet}}$. Reactions were incubated for $20 \mathrm{~min}$ at $37^{\circ} \mathrm{C}$. Note that all concentrations indicated represent final concentrations after rapid mixing with $50 \mathrm{~S}$ subunits.

smFRET experiments. 30S ICs were tethered to the polyethylene glycol (PEG)/ biotinylated-PEG-derivatized surface of a quartz microfluidic flowcell via a biotinstreptavidin-biotin bridge and imaged it at single-molecule resolution using a labbuilt, wide-field, prism-based TIRF microscope. The $\mathrm{Cy} 3$ fluorophore was directly excited using a 532-nm, diode-pumped, solid-state laser (CrystaLaser) under a $\sim 12 \mathrm{~mW}$ excitation laser power, as measured at the prism. The Cy3 and $\mathrm{Cy} 5$ fluorescence emissions were simultaneously collected by a 1.2 numerical aperture/ $\times 60$ water-immersion objective (Nikon) and wavelength separated using a two channel, simultaneous-imaging system (DV2, Photometrics Inc.). The Cy3 and Cy5 fluorescence emissions were imaged at an acquisition time of $0.1 \mathrm{~s}$ per frame using a $512 \times 512$ pixel, back-illuminated EMCCD camera (Cascade II:512, Photometrics Inc.) operating with 2 pixel $\times 2$ pixel binning. As before ${ }^{28}$, we supplemented all buffers with $1 \mathrm{mM}$ GTP, $\sim 1 \mu \mathrm{M}$ IF1, and $25 \mathrm{nM}$ wtIF2 [Cy5] $]_{\mathrm{dIV}}$, mutIF2 $[\mathrm{Cy} 5]_{\mathrm{dIV}}$, or wtIF2 $[\mathrm{Cy} 5]_{\mathrm{dIII}}$ in order to allow these IFs to re-associate with $30 \mathrm{~S}$ ICs from which they might have dissociated during tethering and/or TIRF imaging.

The Cy3 and Cy5 fields of view from each individual movie were aligned using custom-built software written in Java, and co-localized Cy3 and Cy5 fluorescence signals were used to plot raw, $\mathrm{Cy} 3$ and $\mathrm{Cy} 5$, fluorescence intensity vs. time trajectories. The $\mathrm{Cy} 5$ fluorescence intensity in these trajectories was corrected for bleed-through of $\mathrm{Cy} 3$ emission into the Cy5 channel ( 7\%) and the trajectories were baseline-corrected using custom scripts written in MATLAB. The raw, bleedthrough- and baseline-corrected $\mathrm{Cy} 3$ and $\mathrm{Cy} 5$ fluorescence intensity vs. time trajectories that exhibited anti-correlation of the $\mathrm{Cy} 3$ and Cy5 fluorescence signals and either (i) single-step photobleaching of the Cy3 and/or Cy5 fluorophores, or (ii) average Cy3 and Cy5 fluorescence intensities characteristic of single Cy3 and Cy5 fluorophores, were kept for further analysis. $E_{\mathrm{FRET}}$ vs. time trajectories (hereafter $E_{\text {FRET }}$ trajectories) were obtained from the raw, bleed-through- and baseline-corrected Cy 3 and Cy5 trajectories by calculating the $E_{\mathrm{FRET}}$ value at each time point of the trajectory. The $E_{\mathrm{FRET}}$ values were calculated by dividing the $\mathrm{Cy} 5$ fluorescence intensity $\left(I_{\mathrm{Cy} 5}\right)$ by the sum of the $\mathrm{Cy} 3$ and $\mathrm{Cy} 5$ fluorescence intensities $\left(I_{\mathrm{CY} 5+} I_{\mathrm{Cy} 3}\right)$. The $E_{\mathrm{FRET}}$ trajectories were truncated after photobleaching of Cy3.

Kinetic parameters describing the interaction of IF2 with the 30S IC was extracted from the $E_{\mathrm{FRET}}$ trajectories using a previously described ${ }^{28}$ transition probability matrix-based population decay analysis. The $E_{\mathrm{FRET}}$ trajectories from three independent data sets were idealized to a HMM using the vbFRET software package ${ }^{40}$. Subsequently, the idealized $E_{\mathrm{FRET}}$ trajectories were used to construct a $2 \times 2$ counting matrix with matrix elements $n_{i j}$, where $i$ represents the IF2-free state of the 30S IC $\left(E_{\mathrm{FRET}} \leq 0.2\right)$ and $j$ represents the IF2-bound state of the 30S IC $\left(E_{\mathrm{FRET}}>0.2\right)$. The row elements of the counting matrix were normalized to construct a $2 \times 2$ transition probability matrix with matrix elements $\mathrm{p}_{i j}$. The diagonal elements of the transition probability matrix $\left(\mathrm{p}_{i i}\right.$ and $\mathrm{p}_{i j}$ ) report the probability of staying in the IF2-free state $\left(\mathrm{p}_{i i}\right)$ of the 30S IC and the probability of staying in the IF2-bound state $\left(\mathrm{p}_{j j}\right)$ of the $30 \mathrm{~S}$ IC, given a discreet time interval $(\tau)$, which is set by the time resolution of our smFRET experiments ( $0.1 \mathrm{~s}$ per frame) The state transition probabilities, $\mathrm{p}_{i i}$ and $\mathrm{p}_{i j}$, were converted to the rate of IF2 association $\left(k_{a}\right)$ to the 30 S IC and the rate of IF2 dissociation $\left(k_{d}\right)$ from the 30S IC, respectively, using the following two equations:

$$
k_{a}=-\ln \left(p_{\mathrm{IF} 2-\text { free } \rightarrow \mathrm{IF} 2-\text { free }}\right) /[\mathrm{IF} 2] t \text { and } k_{d}=-\ln \left(p_{\mathrm{IF} 2-\text { bound } \rightarrow \mathrm{IF} 2-\text { bound }}\right) / t
$$

The transition probability matrix-based population decay analysis described in the previous paragraph was used to correct for the effects of $\mathrm{Cy} 5$ photobleaching in the $E_{\mathrm{FRET}}$ trajectories from $30 \mathrm{~S} \mathrm{IC}_{\mathrm{wT}}$ and $30 \mathrm{SIC}_{\mathrm{mT}}$ and to correct for the effects of Cy3 photobleaching in the $E_{\mathrm{FRET}}$ trajectories from $30 \mathrm{~S}_{\mathrm{IC}} \mathrm{C}_{\mathrm{w}, \mathrm{Met}}$ and $30 \mathrm{~S} \mathrm{IC}_{\mathrm{wT}, \mathrm{OH}}$. The exceedingly stable and long-lived binding of wtIF2 and mutIF2 to $30 \mathrm{~S} \mathrm{IC}_{\mathrm{wT}}$ and $30 \mathrm{SIC}_{\mathrm{mT}}$ (Fig. 1a, b) results in a significant number of $E_{\mathrm{FRET}}$ trajectories in which IF2 remains stably bound to the 30S IC after Cy5 photobleaching. The failure to account for this in these $E_{\mathrm{FRET}}$ trajectories results in an aberrantly high probability of remaining in the IF2-free state and thus a significant overestimation of $k_{\mathrm{d}}$. Therefore, to correct for the effects of Cy5 photobleaching on $k_{\mathrm{d}}$, the final dwell of each of the $E_{\mathrm{FRET}}$ trajectories that exhibited an $E_{\mathrm{FRET}} \leq 0.2$ was not included in our analysis.

On the other hand, the exceedingly rare and short-lived transitions to the IF2bound state that are observed for the interaction of wtIF2(GTP) with pseudo 30S ICs (30S IC $\mathrm{wT}_{\mathrm{w}, \mathrm{Met}}$ and $30 \mathrm{SIC}_{\mathrm{wT}, \mathrm{OH}}, \mathrm{Fig}$. 3a and b, respectively), result in a subpopulation of $E_{\mathrm{FRET}}$ trajectories originating from pseudo 30S ICs that are capable of undergoing an IF2 binding event, but fail to do so during our observation window. The failure to account for this subpopulation of $E_{\mathrm{FRET}}$ trajectories, which represent long dwells comprised of transitions in the IF2-free state of the 30S IC, results in a decrease in the probability of remaining in the IF2free state and thus an overestimation of $k_{\mathrm{a}}$. To calculate corrected values of $k_{\mathrm{a}}$ for $30 \mathrm{IC}_{\mathrm{wT}, \mathrm{Met}}$ and $30 \mathrm{~S} \mathrm{IC}_{\mathrm{wT}, \mathrm{OH}}$, we generated a series of simulated $E_{\mathrm{FRET}}$ trajectories with an $E_{\mathrm{FRET}}$ value of zero and pooled these with the observed $E_{\mathrm{FRET}}$ trajectories. 
The number of simulated $E_{\mathrm{FRET}}$ trajectories for each pseudo 30S IC was determined by taking the number of observed $E_{\mathrm{FRET}}$ trajectories that exhibited at least one IF2 binding event in $30 \mathrm{~S}^{\mathrm{IC}_{\mathrm{wT}, \mathrm{Met}}}$ or $30 \mathrm{~S}_{\mathrm{IC}} \mathrm{C}_{\mathrm{wT}, \mathrm{OH}}$ and multiplying by a correction factor given by:

$$
\text { Correction factor }(\mathrm{Cf})=\frac{\% \text { capable }-\% \text { observed }}{\% \text { observed }},
$$

where "\% capable" represents the fraction of $E_{\mathrm{FRET}}$ trajectories that exhibited at least one binding event in the analogous 30S IC formed with fMet-tRNA ${ }^{\text {fMet }}$ (30S $\mathrm{IC}_{\mathrm{wT}}$ ), and "\% observed" represents the fraction of $E_{\mathrm{FRET}}$ trajectories that exhibited at least one binding event in $30 \mathrm{SI}_{\mathrm{wT}, \mathrm{Met}}$ or $30 \mathrm{~S} \mathrm{IC}_{\mathrm{wT}, \mathrm{OH}}$. The length of the simulated $E_{\mathrm{FRET}}$ trajectories was set to the average length of the observed $E_{\mathrm{FRET}}$ trajectories for either $30 \mathrm{SIC}_{\mathrm{wT}, \mathrm{Met}}$ or $30 \mathrm{SIC}_{\mathrm{wT}, \mathrm{OH}}$ prior to $\mathrm{Cy} 3$ photobleaching.

Ensemble toeprinting and kinetic experiments. The primer-extension inhibition-, or "toeprinting"-based IF2 activity assay reports on the position of the 30S IC on mRNApri-ext with single-nucleotide resolution and thus measures the ability of IF2 to direct the selection of the authentic initiator fMet-tRNA ${ }^{\mathrm{fMet}}$ into the P site of the 30S IC, over elongator tRNAs. Primer extension was performed using the Avian Myeloblastosis (AMV) reverse transcriptase as described previously 28,39 Briefly, $25 \mu$ l reactions containing $5 \mu$ l of the various 30S ICs, $1.2 \mathrm{mM}$ ATP, $0.5 \mathrm{mM}$ of each dNTP, and 6 units of AMV and Tris-Polymix with $10 \mathrm{mM} \mathrm{Mg}(\mathrm{OAc})_{2}$ were incubated at $37^{\circ} \mathrm{C}$ for $15 \mathrm{~min}$. Following phenol-chloroform extraction and ethanol precipitation, complementary DNA (cDNA) pellets were re-suspended in formamide loading buffer, heat denatured at $95^{\circ} \mathrm{C}$ for $5 \mathrm{~min}$, and cDNA fragments were resolved on a $9 \%$ sequencing gel.

Rayleigh light scattering-based ensemble kinetic 50S subunit joining experiments were performed as described previously ${ }^{21}$. Briefly, $0.6-0.8 \mathrm{ml}$ of 0.36 $\mu \mathrm{M} 50$ S subunits and $0.6-0.8 \mathrm{ml}$ mixture containing $0.36 \mu \mathrm{M}$ 30S IC assembled with IF1, fMet-tRNA ${ }^{\mathrm{fMet}}$, or tRNA ${ }^{\mathrm{fMet}}$ (or no tRNA) and IF2 (wildtype or mutant, added in different concentrations) were pre-incubated for $20 \mathrm{~min}$ at $37^{\circ} \mathrm{C}$ and loaded into the syringes of our stopped-flow instrument (SX-20 Applied Photophysics, Leatherhead, UK). The kinetics of 70S IC formation was monitored at $37^{\circ} \mathrm{C}$ with light scattering after rapid mixing of equal volumes of the $30 \mathrm{~S} \mathrm{IC}$ mixture and the $50 \mathrm{~S}$ subunits.

Data availability. The data that support the findings of this study are available from the corresponding author upon request.

Received: 19 January 2017 Accepted: 21 September 2017

Published online: 14 November 2017

\section{References}

1. Tsai, A. et al. Heterogeneous pathways and timing of factor departure during translation initiation. Nature 487, 390-393 (2012).

2. Milón, P., Maracci, C., Filonava, L., Gualerzi, C. O. \& Rodnina, M. V. Real-time assembly landscape of bacterial $30 \mathrm{~S}$ translation initiation complex. Nat. Struct. Mol. Biol. 19, 609-615 (2012).

3. Hartz, D., McPheeters, D. S. \& Gold, L. Selection of the initiator tRNA by Escherichia coli initiation factors. Genes Dev. 3, 1899-1912 (1989).

4. Antoun, A., Pavlov, M. Y., Lovmar, M. \& Ehrenberg, M. How initiation factors tune the rate of initiation of protein synthesis in bacteria. EMBO J. 25, 2539-2550 (2006).

5. Antoun, A., Pavlov, M. Y., Lovmar, M. \& Ehrenberg, M. How initiation factors maximize the accuracy of tRNA selection in initiation of bacterial protein synthesis. Mol. Cell 23, 183-193 (2006).

6. Milon, P. et al. The ribosome-bound initiation factor 2 recruits initiator tRNA to the 30S initiation complex. EMBO Rep. 11, 312-316 (2010).

7. Caban, K. \& Gonzalez, R. L. The emerging role of rectified thermal fluctuations in initiator aa-tRNA- and start codon selection during translation initiation. Biochimie 114, 30-38 (2015).

8. Grigoriadou, C., Marzi, S., Pan, D., Gualerzi, C. O. \& Cooperman, B. S. The translational fidelity function of IF3 during transition from the $30 \mathrm{~S}$ initiation complex to the 70S initiation complex. J. Mol. Biol. 373, 551-561 (2007).

9. Grigoriadou, C., Marzi, S., Kirillov, S., Gualerzi, C. O. \& Cooperman, B. S. A quantitative kinetic scheme for $70 \mathrm{~S}$ translation initiation complex formation. $J$. Mol. Biol. 373, 562-572 (2007).

10. Milon, P., Konevega, A. L., Gualerzi, C. O. \& Rodnina, M. V. Kinetic checkpoint at a late step in translation initiation. Mol. Cell 30, 712-720 (2008).

11. MacDougall, D. D. \& Gonzalez, R. L. Translation initiation factor 3 regulates switching between different modes of ribosomal subunit joining. J. Mol. Biol. 427, 1801-1818 (2015).
12. Marshall, R. A., Aitken, C. E. \& Puglisi, J. D. GTP hydrolysis by IF2 guides progression of the ribosome into elongation. Mol. Cell 35, 37-47 (2009).

13. Goyal, A., Belardinelli, R., Maracci, C., Milón, P. \& Rodnina, M. V. Directional transition from initiation to elongation in bacterial translation. Nucleic Acids Res. 43, 10700-10712 (2015)

14. Ling, C. \& Ermolenko, D. N. Initiation factor 2 stabilizes the ribosome in a semirotated conformation. Proc. Natl Acad. Sci. USA 112, 15874-15879 (2015).

15. Guenneugues, M. et al. Mapping the fMet-tRNA(f)(Met) binding site of initiation factor IF2. EMBO J. 19, 5233-5240 (2000).

16. Simonetti, A. et al. Structure of the $30 \mathrm{~S}$ translation initiation complex. Nature 455, 416-420 (2008).

17. Julián, P. et al. The cryo-EM structure of a complete $30 \mathrm{~S}$ translation initiation complex from Escherichia coli. PLoS Biol. 9, e1001095 (2011).

18. Hussain, T., Llácer, J. L., Wimberly, B. T., Kieft, J. S. \& Ramakrishnan, V. Largescale movements of IF3 and tRNA during bacterial translation initiation. Cell 167, 133-144.e13 (2016).

19. Antoun, A., Pavlov, M. Y., Tenson, T. \& Ehrenberg, M. Ribosome formation from subunits studied by stopped-flow and Rayleigh light scattering. Biol. Proced. Online 6, 35-54 (2004).

20. Zorzet, A., Pavlov, M. Y., Nilsson, A. I., Ehrenberg, M. \& Andersson, D. I. Error-prone initiation factor 2 mutations reduce the fitness cost of antibiotic resistance. Mol. Microbiol. 75, 1299-1313 (2010).

21. Pavlov, M. Y., Zorzet, A., Andersson, D. I. \& Ehrenberg, M. Activation of initiation factor 2 by ligands and mutations for rapid docking of ribosomal subunits. EMBO J. 30, 289-301 (2011).

22. Roll-Mecak, A., Cao, C., Dever, T. E. \& Burley, S. K. X-Ray structures of the universal translation initiation factor IF2/eIF5B: conformational changes on GDP and GTP binding. Cell 103, 781-792 (2000).

23. Gualerzi, C. O., Severini, M., Spurio, R., La Teana, A. \& Pon, C. L. Molecular dissection of translation initiation factor IF2. Evidence for two structural and functional domains. J. Biol. Chem. 266, 16356-16362 (1991).

24. Mortensen, K. K. et al. A six-domain structural model for Escherichia coli translation initiation factor IF2. Characterisation of twelve surface epitopes. Biochem. Mol. Biol. Int. 46, 1027-1041 (1998).

25. Rasmussen, L. C. et al. Solution structure of C-terminal Escherichia coli translation initiation factor IF2 by small-angle X-ray scattering. Biochemistry 47, 5590-5598 (2008).

26. Vohlander Rasmussen Louise Carœand Oliveira, C. L. P., Pedersen, J. S., Sperling-Petersen, H. U. \& Mortensen, K. K. Structural transitions of translation initiation factor IF2 upon GDPNP and GDP binding in solution. Biochemistry 50, 9779-9787 (2011).

27. Simonetti, A. et al. Involvement of protein IF2 N domain in ribosomal subunit joining revealed from architecture and function of the full-length initiation factor. Proc. Natl Acad. Sci. USA 110, 15656-15661 (2013).

28. Wang, J., Caban, K. \& Gonzalez, R. L. Ribosomal initiation complex-driven changes in the stability and dynamics of initiation factor 2 regulate the fidelity of translation initiation. J. Mol. Biol. 427, 1819-1834 (2015).

29. Gualerzi, C., Risuleo, G. \& Pon, C. Mechanism of the spontaneous and initiation factor 3-induced dissociation of 30S aminoacyl-tRNA polynucleotide ternary complexes. J. Biol. Chem. 254, 44-49 (1979).

30. Murphy, M. C., Rasnik, I., Cheng, W., Lohman, T. M. \& Ha, T. Probing singlestranded DNA conformational flexibility using fluorescence spectroscopy. Biophys. J. 86, 2530-2537 (2004).

31. Myasnikov, A. G. et al. Conformational transition of initiation factor 2 from the GTP- to GDP-bound state visualized on the ribosome. Nat. Struct. Mol. Biol. 12, 1145-1149 (2005).

32. Allen, G. S., Zavialov, A., Gursky, R., Ehrenberg, M. \& Frank, J. The cryo-EM structure of a translation initiation complex from Escherichia coli. Cell 121, 703-712 (2005).

33. Sprink, T. et al. Structures of ribosome-bound initiation factor 2 reveal the mechanism of subunit association. Sci. Adv. 2, e1501502 (2016).

34. Wienk, H. et al. Structural dynamics of bacterial translation initiation factor IF2. J. Biol. Chem. 287, 10922-10932 (2012).

35. Huang, C., Mandava, C. S. \& Sanyal, S. The ribosomal stalk plays a key role in IF2-mediated association of the ribosomal subunits. J. Mol. Biol. 399, 145-153 (2010).

36. Naaktgeboren, N., Schrier, P., Möller, W. \& Voorma, H. O. The involvement of protein L11 in the joining of the 30-S initiation complex to the 50-S subunit. Eur. J. Biochem. 62, 117-123 (1976).

37. Simonetti, A. et al. Structure of the protein core of translation initiation factor 2 in apo, GTP-bound and GDP-bound forms. Acta Crystallogr. D Biol. Crystallogr. 69, 925-933 (2013).

38. Antoun, A., Pavlov, M. Y., Andersson, K., Tenson, T. \& Ehrenberg, M. The roles of initiation factor 2 and guanosine triphosphate in initiation of protein synthesis. EMBO J. 22, 5593-5601 (2003). 
39. Fei, J. et al. A highly purified, fluorescently labeled in vitro translation system for single-molecule studies of protein synthesis. Methods Enzymol. 472, 221-259 (2010).

40. Bronson, J. E., Fei, J., Hofman, J. M., Gonzalez, R. L. \& Wiggins, C. H. Learning rates and states from biophysical time series: a Bayesian approach to model selection and single-molecule FRET data. Biophys. J. 97, 3196-3205 (2009).

\section{Acknowledgements}

We thank D. MacDougall, C. Kinz-Thompson, and B. Huang for insightful discussions and advice; we would also like to thank J. Wang for developing the IF2-tRNA smFRET signal and for providing Cy3-labeled tRNAs. This work was supported by funds to R.L.G. from the National Institutes of Health (R01 GM 084288). K.C. was supported by an American Cancer Society postdoctoral fellowship (125201).

\section{Author contributions}

K.C., M.P., M.E., and R.L.G. designed the research; K.C. performed the smFRET experiments and M.P. performed the ensemble rapid kinetic light scattering experiments; K.C., M.P., M.E., and R.L.G. analyzed the data; K.C., M.P., M.E., and R.L.G. wrote the manuscript; all four authors approved the final manuscript.

\section{Additional information}

Supplementary Information accompanies this paper at doi:10.1038/s41467-017-01492-6.
Competing interests: The authors declare no competing financial interests.

Reprints and permission information is available online at http://npg.nature.com/ reprintsandpermissions/

Publisher's note: Springer Nature remains neutral with regard to jurisdictional claims in published maps and institutional affiliations.

(c) Open Access This article is licensed under a Creative Commons Attribution 4.0 International License, which permits use, sharing, adaptation, distribution and reproduction in any medium or format, as long as you give appropriate credit to the original author(s) and the source, provide a link to the Creative Commons license, and indicate if changes were made. The images or other third party material in this article are included in the article's Creative Commons license, unless indicated otherwise in a credit line to the material. If material is not included in the article's Creative Commons license and your intended use is not permitted by statutory regulation or exceeds the permitted use, you will need to obtain permission directly from the copyright holder. To view a copy of this license, visit http://creativecommons.org/ licenses/by/4.0/.

(C) The Author(s) 2017 